\title{
Dynamics of ordering processes in annealed dilute systems: Island formation, vacancies at domain boundaries, and compactification
}

Shah, Peter Jivan; Mouritsen, Ole G.

Published in:

Physical Review B

Link to article, DOI:

10.1103/PhysRevB.41.7003

Publication date:

1990

Document Version

Publisher's PDF, also known as Version of record

Link back to DTU Orbit

Citation (APA):

Shah, P. J., \& Mouritsen, O. G. (1990). Dynamics of ordering processes in annealed dilute systems: Island formation, vacancies at domain boundaries, and compactification. Physical Review B, 41(10), 7003-7018. https://doi.org/10.1103/PhysRevB.41.7003

\section{General rights}

Copyright and moral rights for the publications made accessible in the public portal are retained by the authors and/or other copyright owners and it is a condition of accessing publications that users recognise and abide by the legal requirements associated with these rights.

- Users may download and print one copy of any publication from the public portal for the purpose of private study or research.

- You may not further distribute the material or use it for any profit-making activity or commercial gain

- You may freely distribute the URL identifying the publication in the public portal 


\title{
Dynamics of ordering processes in annealed dilute systems: Island formation, vacancies at domain boundaries, and compactification
}

\author{
Peter Jivan Shah and Ole G. Mouritsen \\ Department of Structural Properties of Materials, The Technical University of Denmark, Building 307, \\ DK-2800 Lyngby, Denmark
}

(Received 19 May 1989)

\begin{abstract}
The dynamics of the ordering processes in two-dimensional lattice models with annealed vacancies and nonconserved order parameter is studied as a function of temperature and vacancy concentration by means of Monte Carlo temperature-quenching simulations. The models are Ising antiferromagnets with couplings leading to twofold-degenerate as well as fourfolddegenerate ordering. The models are quenched into a phase separation region, which makes it possible for both types of ordering to observe the following scenario of ordering processes: (i) early-time nucleation and growth of ordered domains, (ii) intermediate-time trapping of the mobile vacancies at the domain boundaries, and (iii) late-time diffusion of vacancies along the domain-boundary network towards the surface. In the case of high dilution, the ordering processes correspond to early-time island formation and late-time coarsening and compactification via coalescence. The domain-size distribution function, which is approximately log-normal, is shown to obey dynamical scaling over a substantial time range for both types of ordering. The growth for the pure systems is found to be described by a power law with the classical growth exponent $n=\frac{1}{2}$. For the dilute systems there is a distinct crossover at late times to a much slower, possibly logarithmic growth mode. These results apply to both types of ordering, suggesting that the effects on ordering dynamics of vacancy diffusion and annealed randomness do not depend on the symmetry of the order parameter. The results of the model study are relevant for the interpretation of experiments on ordering in impure systems and off-stochiometric alloys, grain growth in radiation-damaged materials, and may also shed light on aspects of sintering processes. The finding of a crossover from an algebraic growth law for the pure system to a slower, possibly logarithmic growth behavior in the dilute system is in accordance with recent high-resolution low-energy electron-diffraction experiments on the oxygen ordering on W(112) surfaces doped with nitrogen and time-resolved $x$-ray-scattering studies of the ordering in thin films of $\mathrm{Cu}_{3} \mathrm{Au}$ alloys with extra $\mathrm{Cu}$.
\end{abstract}

\section{INTRODUCTION}

The study of the dynamics of ordering processes in condensed matter ${ }^{1}$ is not only important for materials science applications but of uttermost significance for our understanding of nonequilibrium pattern formation and the evolution of order in systems far from thermodynamic equilibrium. ${ }^{2}$ These are very general phenomena, which often arise in systems which suddenly become subject to alteration in thermodynamic conditions. A typical situation involves a system which is thermally quenched below a phase-transition temperature. The system will then initially be unstable or metastable and start to undergo a spontaneous ordering process, leading first to nucleation and growth of small clusters of ordered domains and later to a coarsening of the domains. Finally, the system will approach its uniformly ordered equilibrium state. In particular, the study of universal aspects of the dynamics of late-stage ordering has been intensified recently, experimentally ${ }^{3-14}$ as well as theoretically, ${ }^{15-59}$ and it has been found that the ordering phenomena display dynamical scaling and can be classified into a few universality classes. These classes are mainly determined by whether or not the order parameter is a conserved quantity. Quantities like ordering degeneracy, ${ }^{24,27,31,32}$ spatial dimension, ${ }^{15,16,32}$ details of the interaction potentials, ${ }^{24,31}$ and whether or not additional conservation laws are operative ${ }^{27,33,34}$ appear to be irrelevant as far as the value of the growth exponent is concerned.

The true asymptotic growth behavior may, however, often be veiled by crossover effects, ${ }^{36}$ e.g., due to low-temperature activated processes ${ }^{24,27,31,37,38}$ or parameter effects related to the details of the interaction potential. ${ }^{24,27,32}$ Possibly more important for many real materials under experimental circumstances is that the growth behavior may be influenced by effects due to nonideal conditions and imperfections, such as impurities, ${ }^{13,39-42}$ vacancies, ${ }^{43-47}$ random couplings ${ }^{48,49}$ or random fields, ${ }^{11,12,50-59}$ and second-phase particles. ${ }^{60}$ All these different circumstances impose some kind of randomness with which the ordering process has to comply, leading to effects which may slow down the growth and eventually pin it, or possibly even lead to a completely different growth mode. Two intensively studied cases are that of random fields ${ }^{11,12,50-59}$ and that of 
quenched (immobile) impurities and vacancies. ${ }^{13,39-46}$ In both cases the growth is found to be slowed down dramatically and there is theoretical evidence that the growth mode becomes logarithmic at late times. ${ }^{42,52}$

The case of annealed (mobile) vacancies or impurities and their effects on ordering dynamics has been studied much less. ${ }^{44-46}$ This is the main topic of the present paper. This case is that of a system with at least two components, and it often involves phase separation dynamics, ${ }^{61}$ depending on the actual phase diagram, i.e., the microscopic interactions with and among the mobile imperfections. Similar to the case of quenched impurities $^{42}$ or random fields,${ }^{52}$ the present case is interesting because it may implicate activated processes by which the impurities interact with the moving domain boundary. ${ }^{46}$ This proves to have a dramatic effect on the growth behavior. The cases of annealed and quenched impurities (or vacancies) differ from the case of the random-field Ising model ${ }^{42}$ in that the randomness in the annealed case does not couple directly to the local order parameter as is the case in the random-field Ising model. This is an important distinction from the point of view of nonequilibrium dynamics. ${ }^{42,62}$

Two-dimensional chemisorbed molecular overlayers on solid surfaces constitute a particularly suitable class of systems for studying fundamental aspects of ordering dynamics, since these systems provide a richness of ordering symmetries and degeneracies. ${ }^{63}$ They are attractive to model by computer-simulation techniques, but it is unfortunately very difficult to obtain reliable time-resolved experimental data from them. ${ }^{3,7}$ Moreover, the kinetics of ordering and growth on surfaces may be strongly influenced by surface inhomogeneities, such as steps and impurities. Only very recently has the first systematic experimental work been reported on the growth kinetics of a chemisorbed overlayer in the presence of impurities. ${ }^{13}$

In this paper we report on a Monte Carlo computersimulation study of a simple site-diluted Ising model with $(p=2)$ - as well as $(p=4)$-fold degenerate antiferromagnetic ordering; cf. Fig. 1. ( $p$ denotes the number of degenerate ordered domains.) The model has a nonconserved order parameter. The symmetries of the ordering are the same as those of atomic oxygen chemisorbed on the (110) and (112) surfaces of tungsten [O/W(110) and $\mathrm{O} / \mathrm{W}(112)]$. A preliminary report on the results for the $p=2$ ordering has already appeared. ${ }^{64}$ These chemisorbed systems are among the few for which timeresolved experimental data are available. ${ }^{4-7}$ In the case of an impurity-modulated adsorbate system, the only one which has been studied systematically until now is $\mathrm{O} / \mathrm{W}(112)$ doped with nitrogen. ${ }^{13}$ The general results of the experimental studies on the pure systems are that close to monolayer coverages, the ordering dynamics for $\mathrm{O} / \mathrm{W}(110)$ and $\mathrm{O} / \mathrm{W}(112)$ is described by an algebraic growth law ${ }^{4-7}$

$$
\bar{R}(T) \sim t^{n}
$$

with $n \simeq 0.50$ for $\mathrm{O} / \mathrm{W}(112)$ and $n \simeq 0.28$ for $\mathrm{O} / \mathrm{W}(110)$.
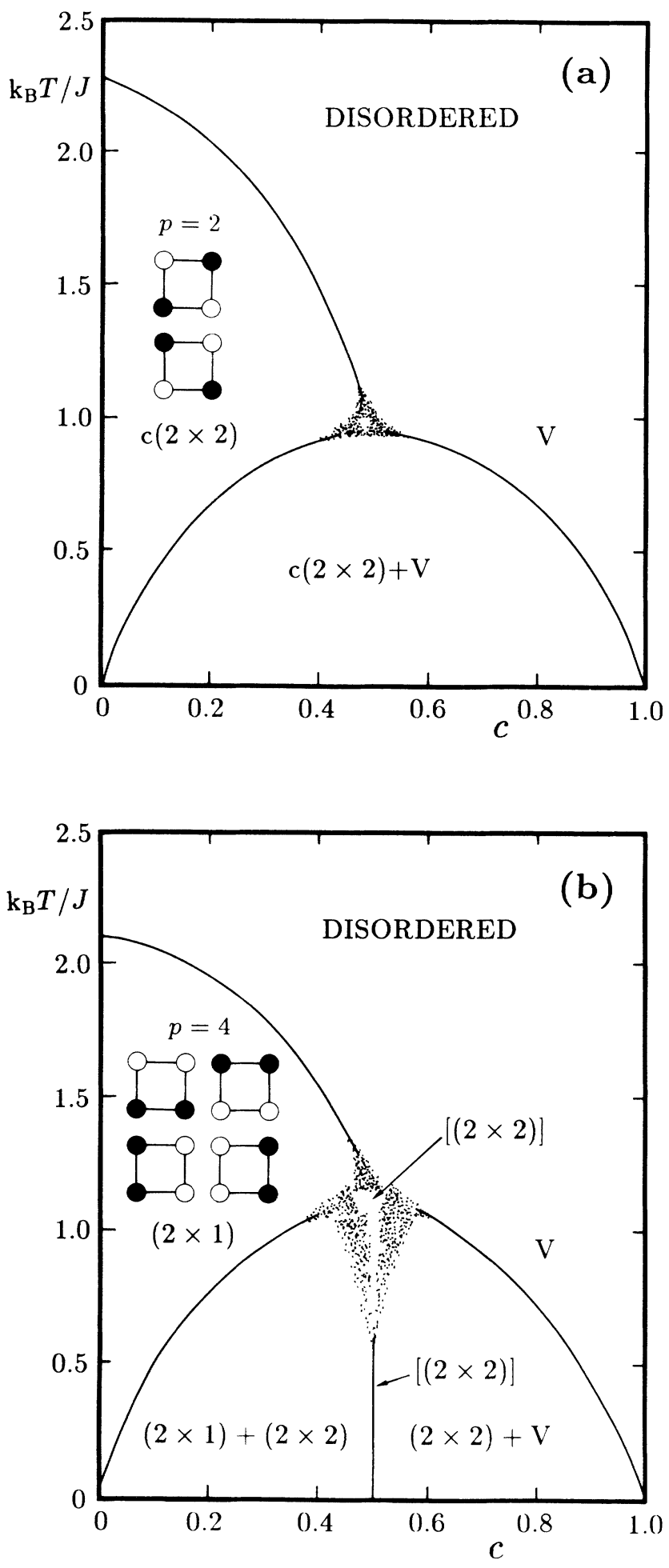

FIG. 1. Phase diagrams (temperature vs vacancy concentration, $c$, and schematic representation of the two types of antiferromagnetic ordering of the Ising model of Eq. (3). (a) $\alpha<\frac{1}{2}$, twofold-degenerate $(p=2)$ antiferromagnetic ordering. (b) $\alpha>\frac{1}{2}$, fourfold-degenerate $(p=4)$ superantiferromagnetic ordering. The hatched areas denote regions of the phase diagrams where the numerical determination of the phase boundaries is uncertain. 
$\bar{R}(t)$ is the characteristic time-dependent linear length scale of the growth process. The first growth-exponent value quoted is in accordance with the classical result, $n=\frac{1}{2}$, from the theories of curvature-driven domain growth by Lifshitz ${ }^{65}$ and Allen and Cahn. ${ }^{66}$ The deviation of the latter result from $n=\frac{1}{2}$ has been interpreted as being either due to a low-temperature crossover effect $^{27}$ or an effect due to a low rate of deexcitation of a precursor state into the chemisorbed state. ${ }^{35}$ Theoretically, it has been shown by computersimulation studies ${ }^{16,18,19,24,27}$ that both ordering degeneracies should lead to the classical growth law with $n=\frac{1}{2}$. Specific calculations of ordering dynamics (with a nonconserved order parameter) in models of $\mathrm{O} / \mathrm{W}(110)$ $(p=4),{ }^{24} \mathrm{O} / \mathrm{W}(112)(p=2),{ }^{16} \mathrm{H} / \mathrm{Fe}(110)(p=2,3),{ }^{22}$ and $\mathrm{O} / \mathrm{Pd}(110)(p=2,3)^{34}$ lead to $n \simeq 0.50$ independent of the value of $p$. For the impure system, Ndoped $\mathrm{O} / \mathrm{W}(112)$, the experimental data obtained by high-resolution low-energy electron-diffraction (LEED) experiments ${ }^{13}$ suggest a dramatic slowing down of the growth rate in a way which is consistent with a logarithmic growth law

$$
\bar{R}(t) \sim(\ln t)^{m}
$$

with $m \simeq 1$. The results of the present model study give evidence of a crossover from a pure-system algebraic growth law with $n=\frac{1}{2}$ to a much slower, possibly logarithmic growth mode for the diluted system, in accordance with the experimental findings.

Regarding ordering dynamics there is a conceptual difference between the chemisorbed systems described above and, e.g., physisorbed and other open systems for which the density is not conserved during the ordering process. For the chemisorbed systems, the density (or total coverage) is constant, although the order parameter of the particular $(2 \times 1)$ ordering is not. Only for $(1 \times 1)$ ordering is the order parameter and the density the same quantity. However, it has been suggested ${ }^{7}$ that for low coverages of $(2 \times 1)$ phases the effect of the antiphase boundaries may be irrelevant, and there is an effective conservation law for the order parameter via the density. This could imply the validity of the Lifshitz-Slyozov mechanism ${ }^{67}$ of growth via evaporationcondensation processes subject to conservation of the order parameter. This issue will also be addressed in this paper.

A number of computer-simulation studies have been carried out on the ordering dynamics in two-dimensional models with quenched randomness, e.g., the randomfield Ising model ${ }^{53-56,59}$ and Ising and Potts models with quenched site dilution. ${ }^{39-41,43-46}$ All these studies suggest a dramatic slowing down (possible logarithmic) of the growth due to the randomness. Very few computersimulation studies have been reported on the effect of annealed site dilution. The first one is the Monte Carlo study by Mouritsen ${ }^{44}$ of orientational pinwheel ordering in models of diatomic molecules $\left(\mathrm{N}_{2}, \mathrm{CO}\right)$ mixed with rotationally inert molecules $(\mathrm{Ar}, \mathrm{Kr})$ physisorbed on graphite. This is a rather special case in which the mobile vacancies (the inert molecules) couple to the order parameter. Subsequently, two Monte Carlo studies appeared on the effect of diffusing vacancies of the ordering in the square Ising ferromagnet. ${ }^{45,46}$ Both of these studies were performed at very low quench temperatures, which led to a pinning of the growth. Ohta et al. ${ }^{47}$ studied by Monte Carlo techniques the domain-boundary morphology in a diluted binary alloy model in tricritical quenches. All these studies showed that, in the case of annealed vacancies and impurities, there is a tendency for vacancy precipitation and trapping of vacancies at the domain boundaries during the growth process.

The study of annealed impurity or vacancy effects is closely related to that of ordering kinetics in offstoichiometric quenches of alloys in which the diffusional motion of the excess atoms couple to the migration of the domain wall. ${ }^{47} \mathrm{~A}$ recent careful time-resolved $\mathrm{x}$-ray study ${ }^{14}$ of the ordering kinetics in $\mathrm{Cu}_{0.79} \mathrm{Au}_{0.21}$ alloys indeed shows a crossover from the pure-system $\left(\mathrm{Cu}_{3} \mathrm{Au}\right)$ algebraic growth law, Eq. (1) with $n \simeq 0.50$, to a logarithmic growth law, Eq. (2), with $m=1-2$.

The layout of the paper is as follows: In Sec. II we introduce the Ising Hamiltonian and its associated microscopic dynamics. Section III deals with computational details, including a description and test of the vectorized dynamics algorithm we have devised and used for the present study. Sections IV and V are devoted to a description of the results obtained for the ordering dynamics of diluted phases with twofold and fourfold degeneracy, respectively. The computer-simulation results are compared with available experimental data in Sec. VI. The paper is concluded in Sec. VII.

\section{MODEL}

\section{A. Hamiltonian and ordering symmetries}

The site-diluted square-lattice spin- $\frac{1}{2}$ Ising antiferromagnet with nearest-neighbor (NN) and next-nearestneighbor (NNN) interactions is described by the Hamiltonian

$$
H=J\left(\sum_{i>j}^{\mathrm{NN}} \sigma_{i} \sigma_{j}+\alpha \sum_{i>j}^{\mathrm{NNN}} \sigma_{i} \sigma_{j}\right)
$$

with $\sigma_{i}=0, \pm 1$. The value $\sigma_{i}=0$ is associated with vacant sites. The global vacancy concentration is $c$. $\alpha$ measures the ratio between NNN and NN coupling strengths. The lattice has free boundaries. We shall focus on the antiferromagnetic case, $J>0$. For the pure system, $c=0$, and for $\alpha<\frac{1}{2}$, this model leads to simple $c(2 \times 2)$ antiferromagnetic order which is twofold degenerate $(p=2)$; cf. Fig. 1. For $\alpha>\frac{1}{2}$, the order is of $(2 \times 1)$ superantiferromagnetic type, which is fourfold degenerate $(p=4)$; cf. Fig. 1. At $\alpha=\frac{1}{2}$ the two types of order are degenerate. The phase diagram of this model as spanned by $\alpha$ and temperature has been worked out by Binder and Landau 
for $c=0 .{ }^{68}$ We have in this paper investigated the model, Eq. (3), for $\alpha=0$ and $\alpha=1$ leading to twofold and fourfold degenerate ordering, respectively. We shall, in the following, refer to these two cases as the " $p=2$ " model and the " $p=4$ " model. The transition temperatures in the thermodynamic limit of the two models for $c=0$ are $k_{B} T_{c} / J \simeq 2.27$ and 2.10 , respectively.

In the case of annealed site dilution, ${ }^{61} c>0$, the system is effectively a two-component system which will phase separate at sufficiently low temperatures. The phase diagrams of the two models are shown in Fig. 1. The diagrams have been determined by Monte Carlo calculations. It is important to notice that annealed sitediluted models for any concentration, $c>0$, in contrast to models of quenched site dilution, will display cooperative behavior and ordering, provided the temperature is sufficiently low. ${ }^{61}$ In the very dilute range this implies segregation of spins into ordered islands, i.e., phase separation. There is a fundamental difference between the phase-separated regions of the two models. The $p=2$ model will simply phase separate into an antiferromagnetically ordered phase with some dilution and an almost pure vacancy phase. In contrast, the $p=4$ model will at $c=50 \%$ support a so-called stoichiometric compound which is a $(2 \times 2)$ superantiferromagnetic phase [in fact a $p(2 \times 2)$ phase] in which the vacancies form a $2 \times 2$ superlattice, which decouples all the $\mathrm{NN}$ bonds and hence releases their inherent frustration. Therefore, in the $p=4$ model below $c=50 \%$, the system phase separates into a diluted $(2 \times 1)$ phase and a $(2 \times 2)$ phase with some excess spins. Above $c=50 \%$, there is phase separation between a diluted $(2 \times 2)$ phase and an almost pure vacancy phase. The case of $c=50 \%$ is therefore a marginal case of the $p=4$ model. In order to avoid effects due to the structure of the $(2 \times 2)$ phase we have in this paper restricted ourselves to studying the $p=4$ model for $c \leq 5 \%$, in which interval it is found that the effects due to the $(2 \times 2)$ ordering can be neglected. It should be noted that the competition between $(2 \times 2)$ and $(2 \times 1)$ ordering in the present model is similar to the competition between herringbone and pinwheel orientational ordering in the annealed site-diluted anisotropic planar rotor model of 3:1 mixtures of diatomic molecules $\left(\mathrm{N}_{2}\right.$ and $\mathrm{CO}$ ) and rare gases physisorbed on graphite. ${ }^{44}$ The ordering dynamics of the $(2 \times 2)$ phases will be described in a separate paper.

\section{B. Model dynamics and annealed randomness}

The ordering processes governed by the Hamiltonian, Eq. (3), are based on a particular choice of microscopic dynamics which involves spin-flip excitations as well as vacancy diffusion. The spin-flip excitations involve single sites, and they do not conserve the antiferromagnetic order parameter. The vacancy diffusion involves exchange of a spin and a vacancy at nearest- or nextnearest-neighbor sites and a possible simultaneous spin flip of the spin involved. The process conserves the global vacancy concentration.

The dynamic model is implemented as follows using standard Monte Carlo Metropolis importance sampling. ${ }^{69}$ A site, $i$, and a $\mathrm{NN}$ or NNN site, $j$, to $i$ are chosen at random and the following possibilities are considered: (i) If $\sigma_{i}=\sigma_{j}=0$, nothing happens. (ii) If $\sigma_{i} \neq 0$ and $\sigma_{j} \neq 0, \sigma_{i}$ is randomly assigned a new value \pm 1 (spin flip) with the standard Metropolis transition probability of a spin flip. (iii) If $\sigma_{i} \neq 0$ and $\sigma_{j}=0$, or $\sigma_{i}=0$ and $\sigma_{j} \neq 0$, the combined process of exchanging the site variables and randomly assigning a value \pm 1 to the site carrying the spin, is performed according to the Metropolis transition probability of the combined process. In any of the three cases (i)-(iii), the time parameter $t$ is incremented by one unit. This corresponds to a particularly simple choice of time-scale ratio for the spin-flip dynamics and the vacancy diffusivity. Obviously, this procedure may easily be generalized to a variable time-scale ratio as in the study by Srolovitz and Hassold. ${ }^{46}$ It is important to point out that for modeling a particular material it may well be necessary to consider a particular timescale ratio. Moreover, it is known that the diffusion rate of vacancies for many materials is strongly dependent on temperature. Again, our model can be extended to account for such complications, provided the temperature variation of the diffusion rate is known or assumed.

The site dilution of the model may be viewed as a kind of randomness with which the system has to comply during the nonequilibrium ordering process. For convenience this may be called annealed randomness to distinguish it from the quenched randomness of random-field Ising models ${ }^{51,52}$ and systems with immobile vacancies or impurities. ${ }^{42}$ In the case of annealed randomness the system has an additional mode of relaxation by way of manipulating the vacancy distribution. In contrast to the random-field Ising model, the model with site dilution considered here does not involve random variables which couple directly to the local order parameter. ${ }^{42}$ This is of importance for assigning the model to a particular dynamical universality class. ${ }^{28}$

\section{COMPUTATIONAL TECHNIQUES}

\section{A. Measures of growth}

The ordering processes are studied as a function of time, $t$, subsequent to a deep thermal quench from a very high temperature to some low temperature, $k_{B} T / J$, in the phase separation region. The time is measured in units of Monte Carlo steps per site (MCS/S). A random configuration is used to represent the high-temperature initial state. After the quench, the system starts to develop domains of ordered spins which grow as a function of time. A domain is defined as a connected region of spins with the same type of ordering. $\mathrm{NN}$ as well as NNN connections are admitted. Whether a spin is associated with a particular type of ordering is decided by a simple majority rule which involves the spin and its four 
nearest neighbors. Other choices of connectivity and domain rules are possible and the particular choice will not affect the large-scale properties of the growth measure.

The simulations are carried out on a variety of different lattice sizes, $N$, in order to estimate finite-size effects. The major part of the results to be reported are obtained for $N=100 \times 100$ and $200 \times 200$. Some quenches have also been performed on smaller lattices in order to demonstrate, for the very high dilution, the late-time compactification process.

The growth of the domains may be monitored using a variety of measures of linear length scale which are all expected to display the same time dependence, provided scaling holds. ${ }^{19}$ In this work we have restricted ourselves to using as a primary measure of length scale the average domain size, $\bar{R}(t)$, determined from the linear-size distribution function, $P(R, t)$,

$$
\bar{R}(t)=\int_{0}^{\infty} R(t) P(R, t) \mathrm{d} R,
$$

where $P(R, t)$ is appropriately normalized, $\int_{0}^{\infty} P(R, t) \mathrm{d} R=1 . P(R, t)$ is determined as an average over an ensemble of size-distribution functions calculated directly from the microconfigurations.

In addition to $\bar{R}(t)$ we have used the excess energy per site

$$
\Delta E(t)=E(t)-E(T)
$$

as a growth measure. $E(T)$ is the equilibrium internal energy of the system at the temperature to which the system is quenched, and $\Delta E(t)$ is therefore the total energy associated with the nonequilibrium interfaces (domain boundaries) formed during the quench. $E(T)$ has to be determined from equilibrium Monte Carlo calculations. For the dilute systems, such calculations are performed by equilibrating an initially compact and approximately circular domain of uniform ordering. If scaling holds, ${ }^{70}$ one has $\bar{R}(t) \sim \Delta E(t)^{-1}$. It is not obvious, however, that this relation holds for dilute systems.

An additional measure of the length scale which we have used for the fourfold degenerate ordering is the Bragg peak intensity

$$
L(t)=\sqrt{\mathrm{N}}\left(\sum_{i=1}^{4} \psi_{i}^{2}(t)\right)^{1 / 2},
$$

which is calculated by means of the global antiferromagnetic order parameter values, $\psi_{i}(t)$, for the four different domains. Since $L(t)$ is not a self-averaging quantity, ${ }^{23}$ and hence is very difficult to determine accurately for low degeneracy, we only use $L(t)$ as a length-scale measure for $p=4$; cf. Ref. 24 .

\section{B. Vectorized dynamics}

We have developed an updating algorithm for the model dynamics which can be implemented on a vector processor. This algorithm is based on a generalized checkerboard principle by which the lattice is subdivided into a number of equivalent interpenetrating square sub- lattices. In order to allow for parallel updating of the sites in each sublattice, it is required that the spins within a sublattice have no direct interaction bonds. For the general Hamiltonian, Eq. (3), this implies a minimum of 16 sublattices. The dynamics of Sec. II B is then implemented by randomly selecting one of the sublattices and simultaneously updating a certain fraction, $f$, of the sites of that sublattice. The choice of $f$ will be discussed later.

Use of vectorized or parallel updating algorithms in the study of time-dependent Monte Carlo processes ${ }^{21,71,72}$ raises some fundamental problems regarding the nature of the discrete time parameter. Obviously, the master equations describing sequential and parallel updating are very different, and there seems to be no way of rigorously proving that they should lead to the same average dynamical evolution of the system. There is now some evidence $^{72}$ that, as far as relaxation out of nonequilibrium is concerned, the Monte Carlo time parameters of different updating algorithms are linearly related as long as these algorithms are built on spatially local decision criteria. An interesting situation of a nonlocal collective Monte Carlo updating algorithm is that of SwendsenWang dynamics, ${ }^{73}$ which leads to exponential relaxation out of nonequilibrium contrary to a power-law relaxation for local dynamics. ${ }^{71}$ In the absence of a rigorous theory it is, however, necessary to test every new vectorized, parallel, or multispin-coding ${ }^{21}$ updating algorithm against well-known conventional single-site updating algorithms applied to the same problem in order to examine the relationship between the time scales.

In the present implementation we have varied the fraction, $f$, of updated sublattice sites ranging from $f$ $=16 / N$ to $f=1$. The lower limit of this range corresponds to conventional random visitation of single lattice sites; the upper limit corresponds to maximum vectorization and simultaneous visitation of all sites in a sublattice. In Fig. 2 are shown, as an example, the data for

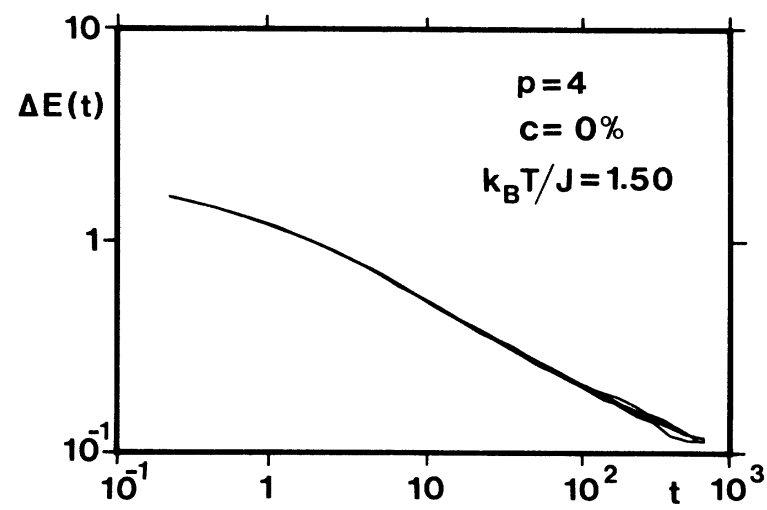

FIG. 2. Log-log plot of the excess energy per spin, $\Delta E(t)$, vs time (in units of MCS/S) for the $p=4$ model on a 100 $\times 100$ lattice at a quench temperature $k_{B} T / J=1.50$ and $c$ $=0 \%$. Data are shown as obtained by a vectorized Monte Carlo checkerboard algorithm which updates a fraction, $f$, of the sites in the 16 sublattices into which the lattice is divided. The curves correspond to $f=16 / 100^{2}, 0.25,0.50,0.75$, and 1. 
$\Delta E(t)$ in the case of $p=4(2 \times 1)$ ordering at $c=0$ for five different values of $f$, ranging from no vectorization to maximum vectorization. It is seen that for all values of $f$, the $\Delta E(t)$ data are accurately represented by the same power law, $\Delta E(t) \sim t^{-n}$, with the expected exponent value, $n \simeq 0.50$. Hence, there is a linear relation between the time scales associated with the different degrees of vectorization. Similar tests leading to the same conclusions have been performed for the $p=2$ case and for $c>0$.

\section{DYNAMICS OF TWOFOLD DEGENERATE ORDERING}

\section{A. Pure system}

The ordering dynamics of the nondiluted simple Ising antiferromagnet $[\alpha=0$ in Eq. (3)] and the thermodynamically equivalent ferromagnet with nonconserved order parameter has been studied in great detail by many authors (Refs. 2, 26, 18, 20, 29, and 37), and there is very strong evidence that these models exhibit dynamical scaling and obey the Allen-Cahn growth law, Eq. (1), with $n=\frac{1}{2}$. We have confirmed this growth law for our free-boundary model as shown in Figs. 3-6 in the case of $\Delta E(t)$ and $\bar{R}(t)$ for different temperatures. The results for $\bar{R}(t)$ display some late-time curvature leading to a slightly lower exponent value. This is a finite-size effect which is enhanced by the strong percolative behavior ${ }^{20}$ of $p=2$ ordering.

The dynamical scaling property of the ordering process is usually demonstrated in the Ising model via the structure factor. ${ }^{16,18}$ Since we have in the present work focused directly on the domain-size distribution function, $P(R, t)$, we present in Fig. 7 the data which show that $P(R, t)$ exhibit dynamical scaling: By introducing the time-dependent scaling variable, $x=R(t) / \bar{R}(t)$, into the distribution function and by renormalizing in order to define the scaling function

$$
\tilde{P}(x)=\bar{R}(t) P(R, t)
$$

[where $\int_{0}^{\infty} \tilde{P}(x) \mathrm{d} x=1$ ], Fig. 7 demonstrates that scaling is fulfilled for $t \geq 80$.

\section{B. Dilute system \\ 1. Island formation, vacancies at domain boundaries, and compactification}

In Fig. 8 are shown typical configurations as they evolve in time for $c=2 \%, 5 \%, 10 \%, 20 \%$, and $50 \%$ in the case of quenches to $k_{B} T / J=0.50$. The striking observation made from this figure is that the vacancies localize themselves at the domain boundaries at a very early time. Simultaneously, vacancies trapped inside the domains tend to precipitate. Large precipitates are found when small domains within large domains dis-

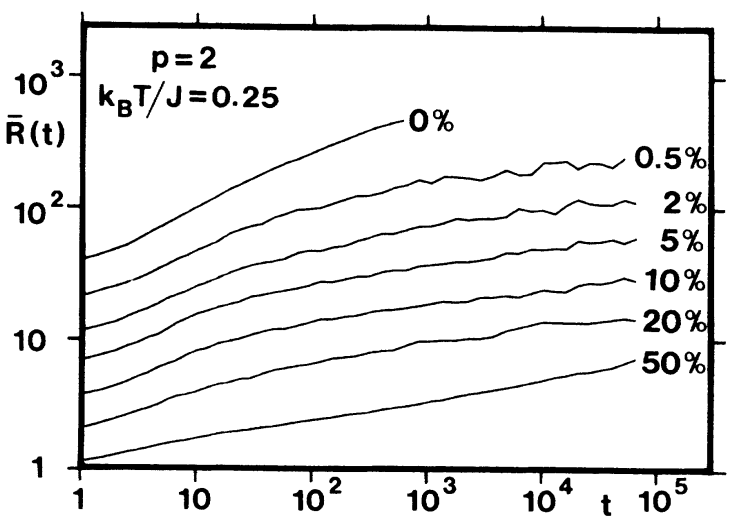

FIG. 3. Log-log plot of the average domain size, $\bar{R}(t)$, vs time $t$ (in units of MCS/S) for quenches to a temperature $k_{B} T / J=0.25$ for different vacancy concentrations, $c$, in the $p=2$ model. For the sake of clarity, the various data sets have been appropriately translated along the vertical axis. The results are derived for systems with $100 \times 100$ and $200 \times 200$ sites.

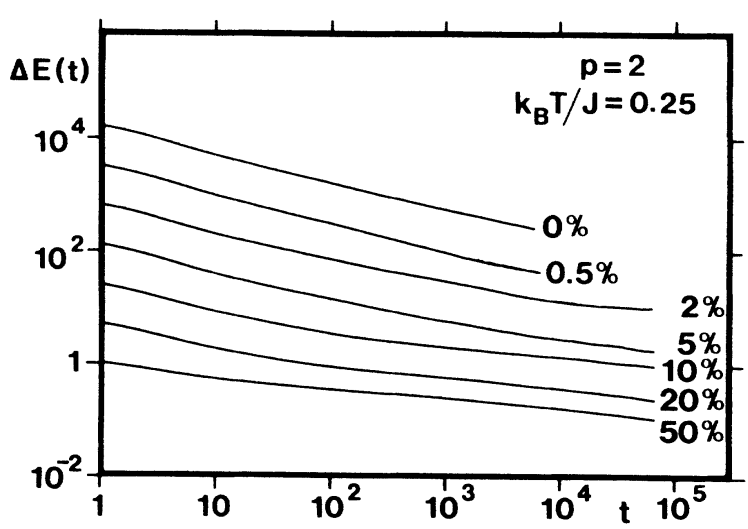

FIG. 4. Same as Fig. 3 but for the excess energy $\Delta E(T)$.

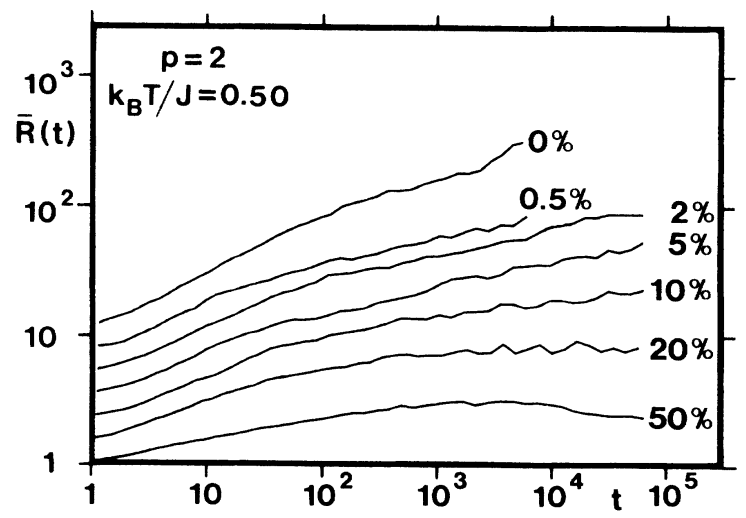

FIG. 5. Same as Fig. 3 but for $k_{B} T / J=0.50$. 


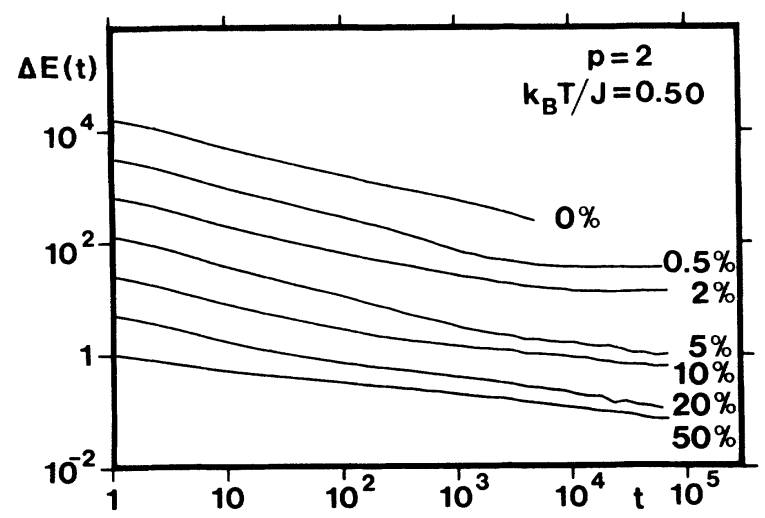

FIG. 6. Same as Fig. 4 but for $k_{B} T / J=0.50$.

appear, leaving their trapped vacancies behind (see, e.g., panels for $c=20 \%$ and $50 \%$ in Fig. 8). Similarly to observations made for the pure system, ${ }^{16}$ finite-size effects at late times may lead to formation of metastable "slab" configurations; cf. Fig. 8 . Figure 9 illustrates for a small system in the very dilute regime the full dynamic scenario of the $p=2$ model from early-time nucleation, island formation, and growth to late-time coarsening of the archipelago of isolated islands.

The snapshots shown in Figs. 8 and 9 give some qualitative insight into the mechanisms of growth and how they are affected by the presence of vacancies. For small degrees of dilution, the late-time growth process proceeds via intermittency of migration of the vacancies along the domain-boundary network towards the surface and curvature-driven domain growth. The accumulation of vacancies in the domain boundaries leads to a

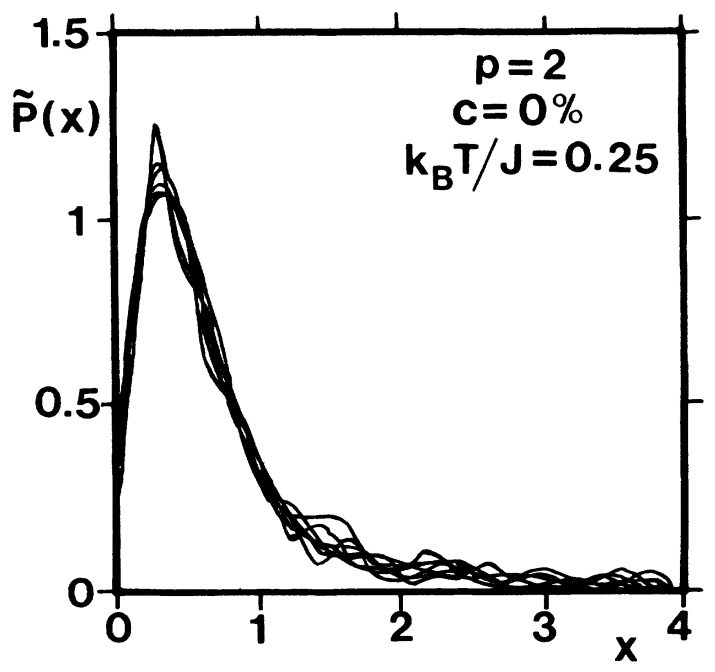

FIG. 7. Dynamical scaling function for the domain-size distribution, $\tilde{P}(x)$ in Eq. (7), for quenches of the $p=2$ model at $c=0 \%$ to a temperature $k_{B} T / J=0.25$. The results, which are given for times $t=79,98,154,194,244,307,386$, and $486 \mathrm{MCS} / \mathrm{S}$, refer to a system with $200 \times 200$ sites. screening of the direct domain-domain interactions and a slowing down of the growth. In the very dilute regime, there is no direct interaction between the various domains, which are only coupled by entropic forces. The final phase-separated equilibrium state is produced via an evaporation-condensation process by which particles from one island migrate to another.

\section{Crossover to a slow growth mode}

The accumulation of vacancies in the domainboundary network during the ordering process, cf. Figs. 8 and 9, leads to a dramatic slowing down of the growth. This is clearly seen in Figs. 4-6. Whereas the early-stage nucleation process is almost as fast as in the pure system, there is a distinct crossover to a slower growth mode at late times. This crossover occurs earlier in time the larger $c$ is. At no stage does the growth become pinned, not even at zero temperature (except slab effects). This is in contrast to previously reported results ${ }^{46}$ for the sitediluted ferromagnet at very low temperatures where the growth stops at late times with a pinned average domain size which decreases as the dilution is increased. The reason for the difference between our results and those of Srolovitz and Hassold ${ }^{46}$ for the ferromagnet is that, whereas we allow for simultaneous Kawasaki and Glauber excitations, Srolovitz and Hassold treat these types of excitations independently. This implies that in their implementation the motion of vacancies trapped at domain-boundary kinks becomes an activated process. We have verified that the diluted ferromagnet indeed becomes unpinned as the temperature is raised.

The vacancy-induced slowing down of the growth is analyzed in Figs. 10 and 11 where semilogarithmic plots of $\bar{R}(t)$ versus $t$ are presented for that data in Figs. 3 and 5. Figures 10 and 11 show that the dilute systems with $c \leq 20 \%$ have an ordering dynamics which is described effectively by a logarithmic growth law

$$
\bar{R}(t) \sim \ln t
$$

for both temperatures. We cannot exclude, on the basis of the present set of data, that the growth behavior at late times, rather than being logarithmic, actually reflects a crossover from the Allen-Cahn law to a slower but still algebraic growth law. For $c=50 \%$, the data at $k_{B} T / J=0.25$ is rather accurately described by a power law, $\bar{R}(t) \sim t^{n}$, with $n \simeq 0.16$. In any case, in the presence of vacancies, even down to $0.5 \%$, the growth is much slower than in the pure system. Since the excess energy, Eq. (5), is not a reliable measure of the linear length scale for dilute systems, we have not performed a similar analysis of the $\Delta E(t)$ data.

Some care should be exercised when comparing data at the same nominal value of $T$ for different values of $c$, since the characteristic temperature scale of the system varies with the dilution. As $c$ is increased, larger thermal fluctuations occur at the same temperature, which 
in turn present greater demands to the statistics of the simulations.

\section{Domain-size distribution and dynamical scaling}

A characterization of the scaling properties of the growth process via the full domain-size distribution func- tion, rather than the average domain size, $\bar{R}(t)$, requires extensive statistics on large systems. Hence, for economical reasons, we have selected a few cases for closer analysis, specifically $c=20 \%$ at $k_{B} T / J=0.50$ and $c=50 \%$ at $k_{B} T / J=0.25$. Characteristic time evolutions in these cases of configurations on a $200 \times 200$ lattice are shown in Fig. 12. Within the time span studied, these two cases

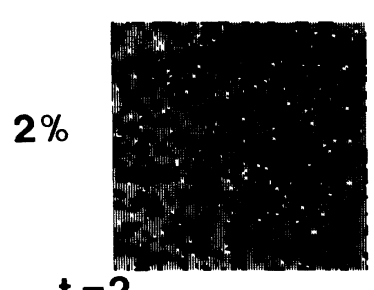

$\mathbf{t}=\mathbf{2}$
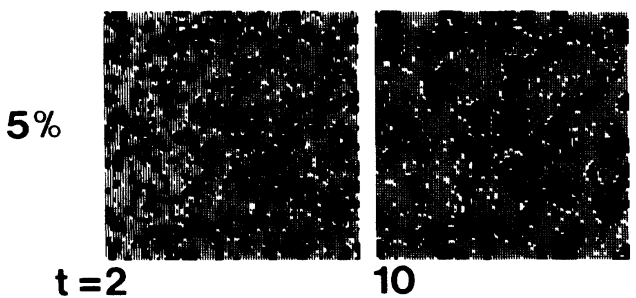

10

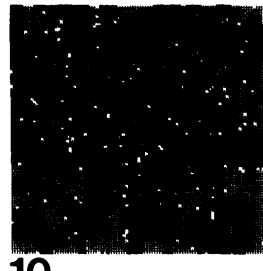

10

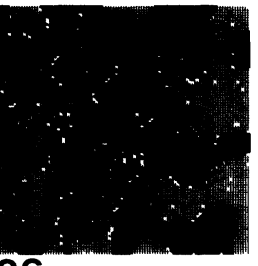

36

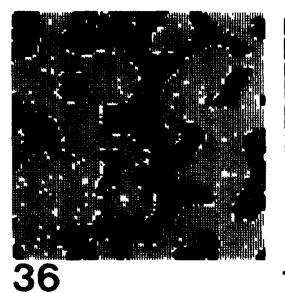

36

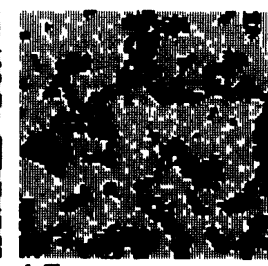

15

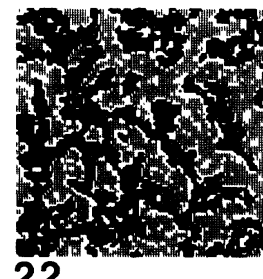

22

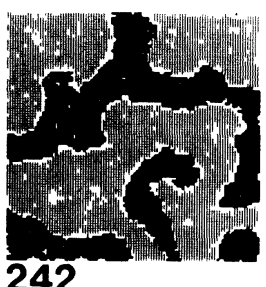

242
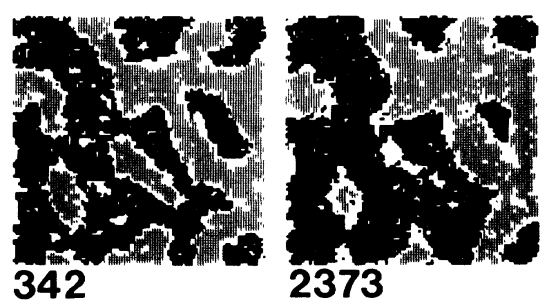

2373

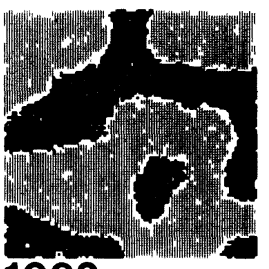

1062

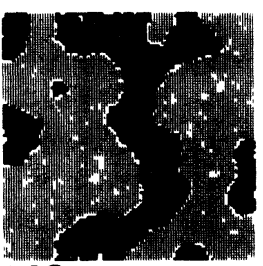

149

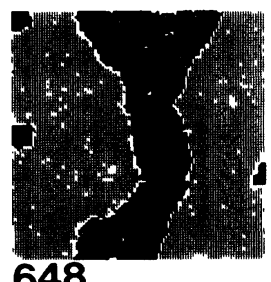

648
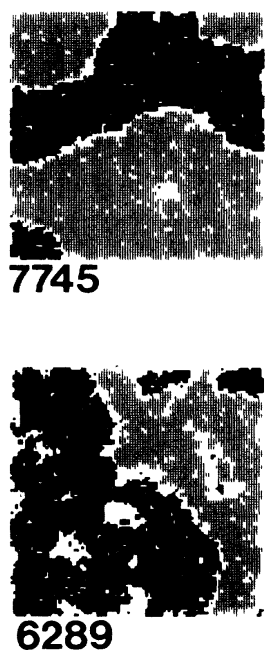

$50 \%$

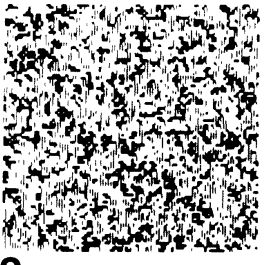

t 2
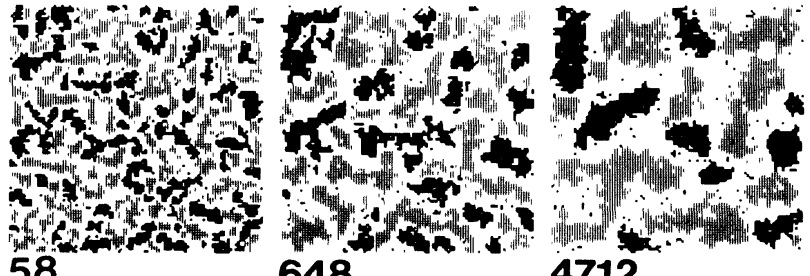

4712

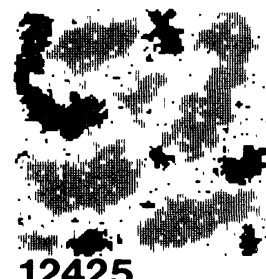

12425

$$
p=2 \quad k_{B} T / J=0.50
$$

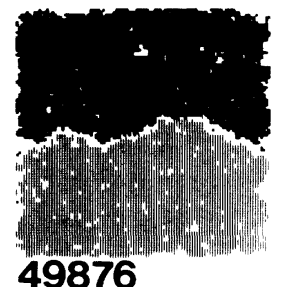

49876
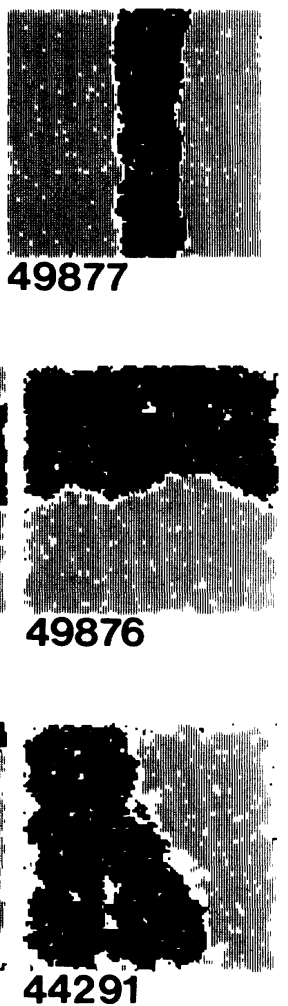

49877

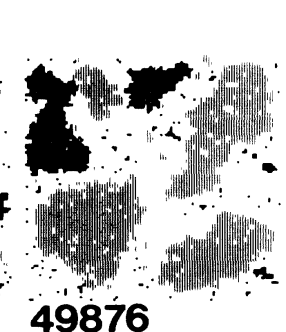

FIG. 8. Snapshots of microconfigurations for the $p=2$ model as they evolve in time $t$ (in units of MCS/S) after quenches to a temperature $k_{B} T / J=0.50$ for vacancy concentrations $c=2 \%, 5 \%, 10 \%, 20 \%$, and $50 \%$. The two types of antiferromagnetically ordered domains are indicated by grey and black regions. The snapshots refer to lattices with $100 \times 100$ sites. 

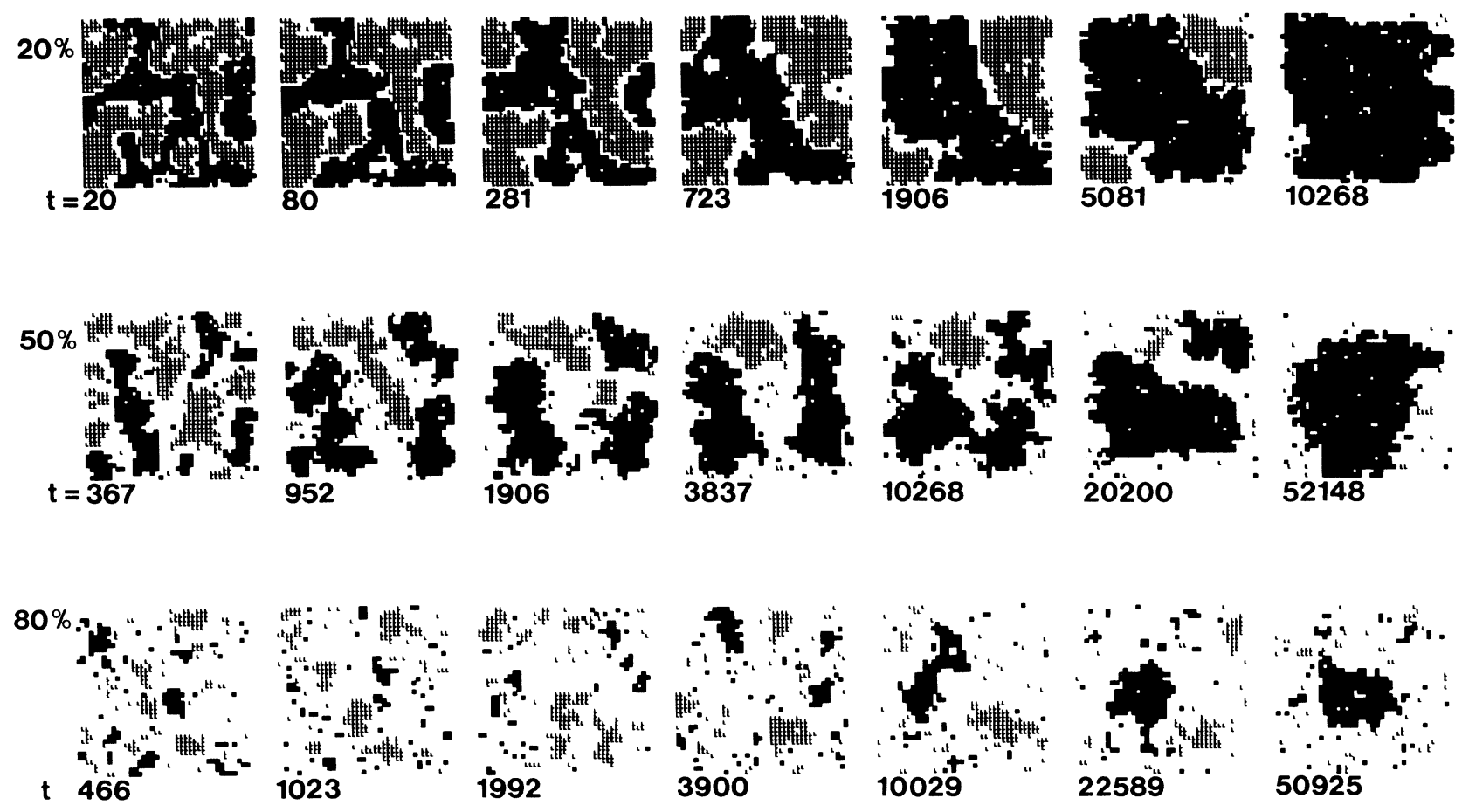

$$
p=2 \quad k_{B} T / J=0.50
$$

FIG. 9. Snapshots of microconfigurations for the $p=2$ model as they evolve in time $t$ (in units of MCS/S) into the equilibrium compact state after quenches to a temperature $k_{B} T / J=0.50$ for high vacancy concentrations, $c=20 \%, 50 \%$, and $80 \%$. The two types of order are indicated by grey and back regions. The configurations refer to a lattice with $40 \times 40$ sites.

are similar with respect to a distinct accumulation of vacancies at the domain boundaries. They differ, however, in the sense that in the case of $c=50 \%$ percolation has not set in.

In Figs. 13 and 14 are shown the domain-size distribution functions in the two cases corresponding to Fig. 12. In both cases, $P(R, t)$ has an asymmetric peak at a finite, temperature-dependent value. The position of the peak moves towards larger values of $R(T)$ as time lapses. The corresponding dynamical scaling functions, $\tilde{P}(x)$ in Eq. (7), are shown as insets in Figs. 13 and 14. It is seen

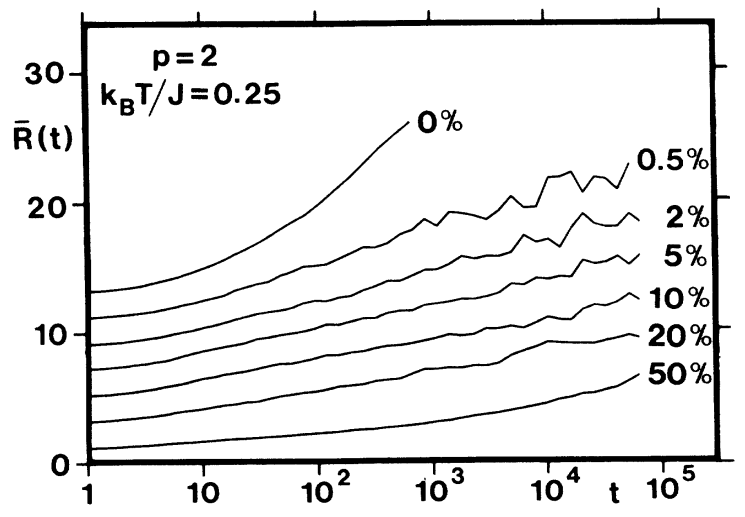

FIG. 10. Semilogarithmic plot of the data in Fig. 3. that the growth process in both cases exhibits dynamical scaling over a substantial range of times.

In both Figs. 13 and $14 P(R, t)$ has a long tail towards large domain sizes making it, as well as the corresponding dynamical scaling function, an approximate log-normal distribution, as demonstrated in Fig. 15 for one of the cases. That the log-normal function is a good representation of domain-growth data has previously been observed ${ }^{32}$ for $p$-state Potts models where $p$ is not too large. Various statistical grain-growth theories have been advanced to explain log normality. ${ }^{74}$

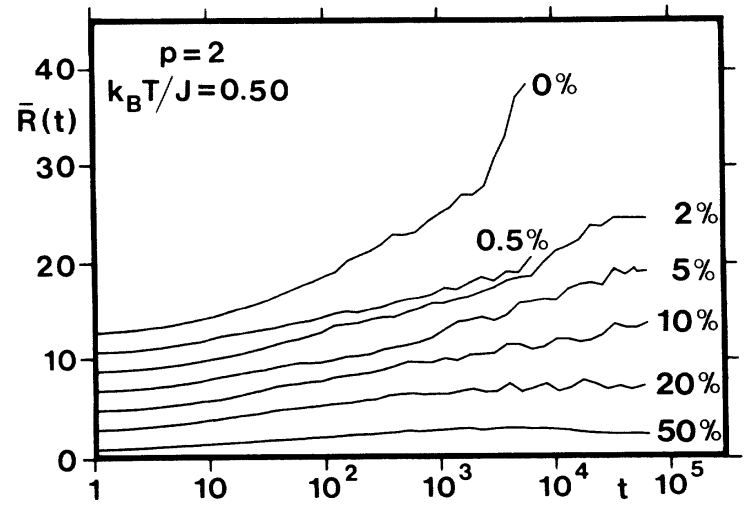

FIG. 11. Semilogarithmic plot of the data in Fig. 5 . 

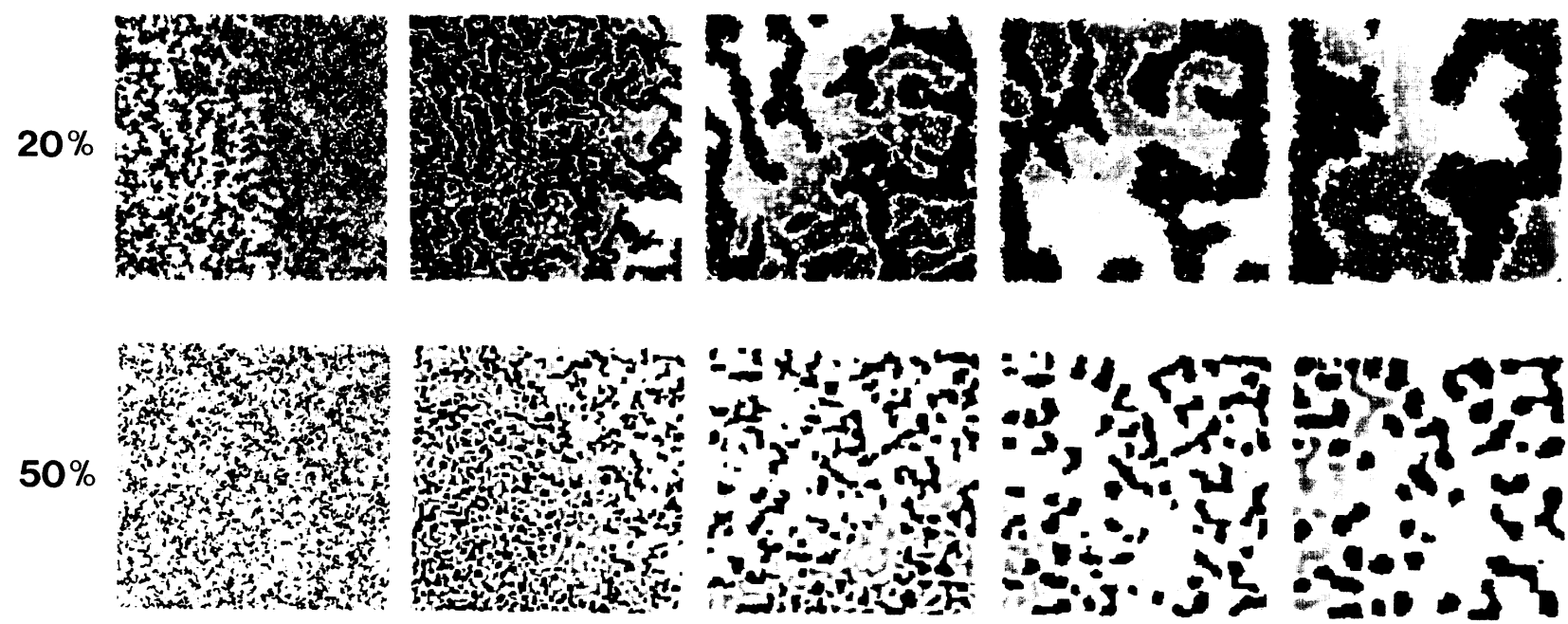

$\mathbf{t}$

5
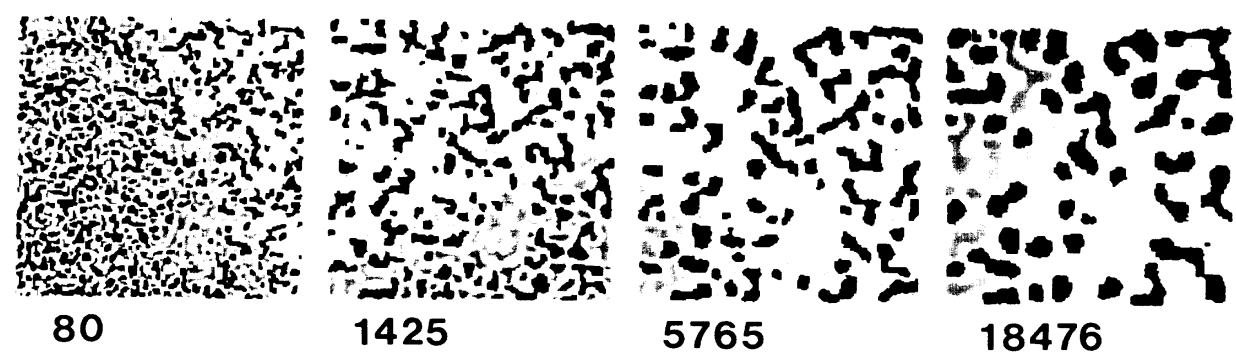

$p=2$

FIG. 12. Snapshots of microconfigurations for the $p=2$ model on a $200 \times 200$ lattice as they evolve in time $t$ (in units of $\mathrm{MCS} / \mathrm{S}$ ) for quenches to a temperature $k_{B} T / J=0.50$ for $c=20 \%$ and a temperature $k_{B} T / J=0.25$ for $c=50 \%$. The two types of antiferromagnetically ordered domains are indicated by grey and black regions.

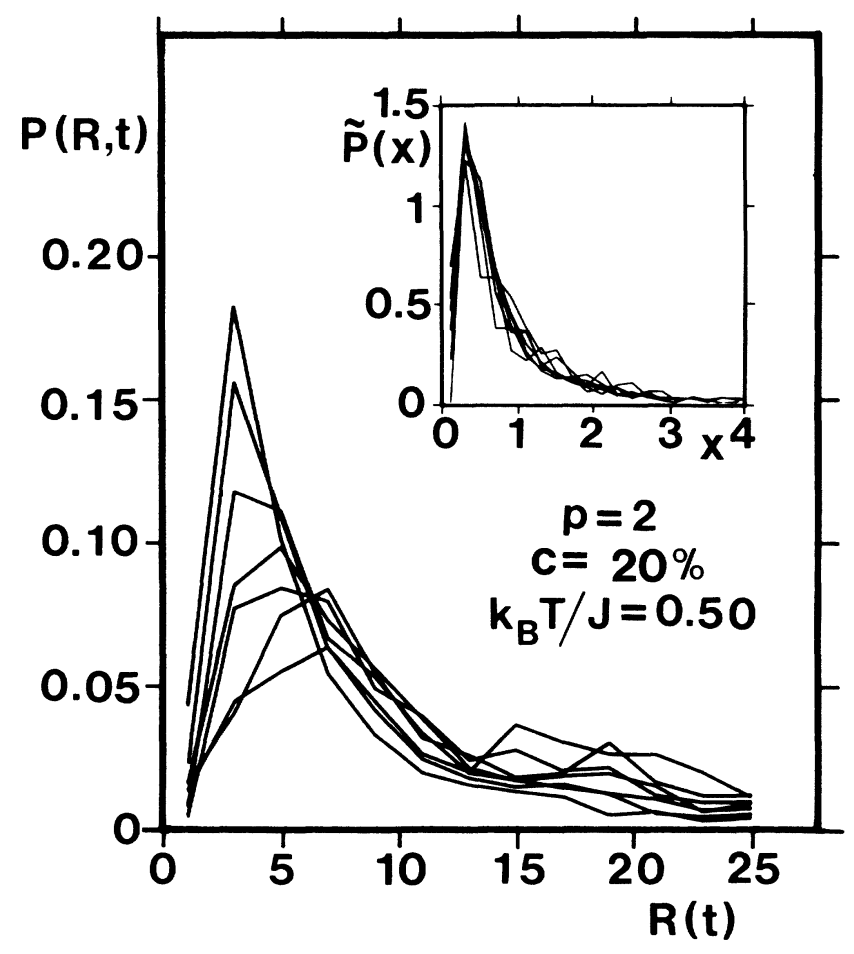

FIG. 13. Domain-size distribution function, $P(R, t)$, for quenches of the $p=2$ model to a temperature $k_{B} T / J=0.50$ for a vacancy concentration $c=25 \%$; cf. Fig. 12 . The maximum of $P(R, t)$, moves to the right as time elapses. Results are given for the following times: $t=4,9,19,38,79,164$, and $340 \mathrm{MCS} / \mathrm{S}$. The inset shows the corresponding dynamic scaling function, $\tilde{P}(x)$ in Eq. (7), where $x=R(t) / \bar{R}(t)$ is the scaling variable.

\section{DYNAMICS OF FOURFOLD-DEGENERATE ORDERING}

Since the analysis and presentation of the computersimulation results for the $p=4$ model with nonconserved order parameter $[\alpha=0$ in Eq. (3)] are very similar to those given in Sec. IV for the $p=2$ model, this section will be very brief, mainly emphasizing the similarities

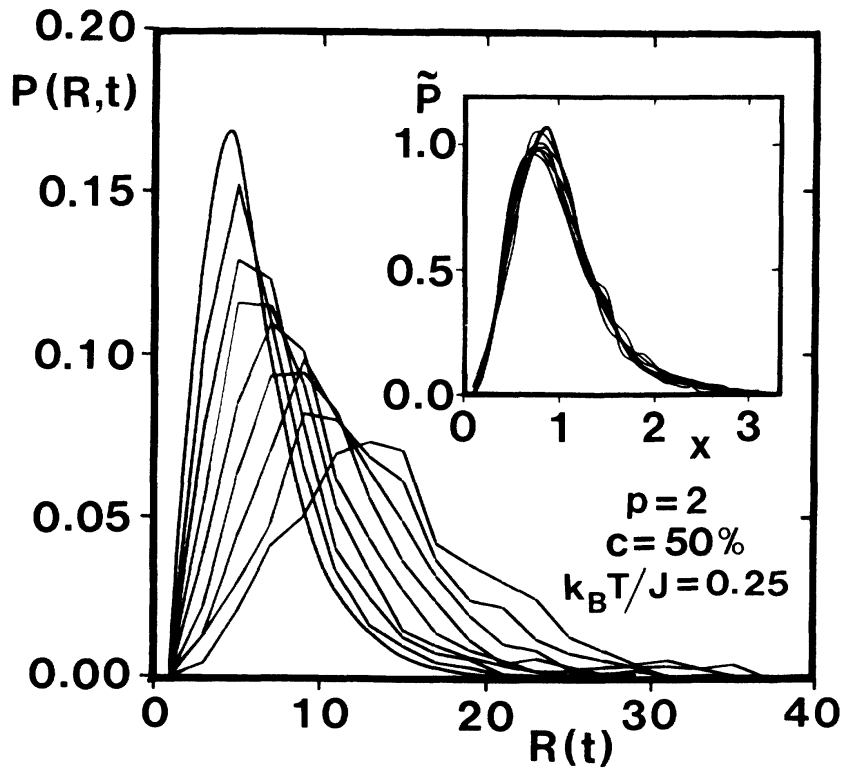

FIG. 14. Same as Fig. 13 for $k_{B} T / J=0.25, c=50 \%$. Results are given for the following times: $t=79,164,340$, $708,1424,2853,5765,11764$, and $18475 \mathrm{MCS} / \mathrm{S}$. 


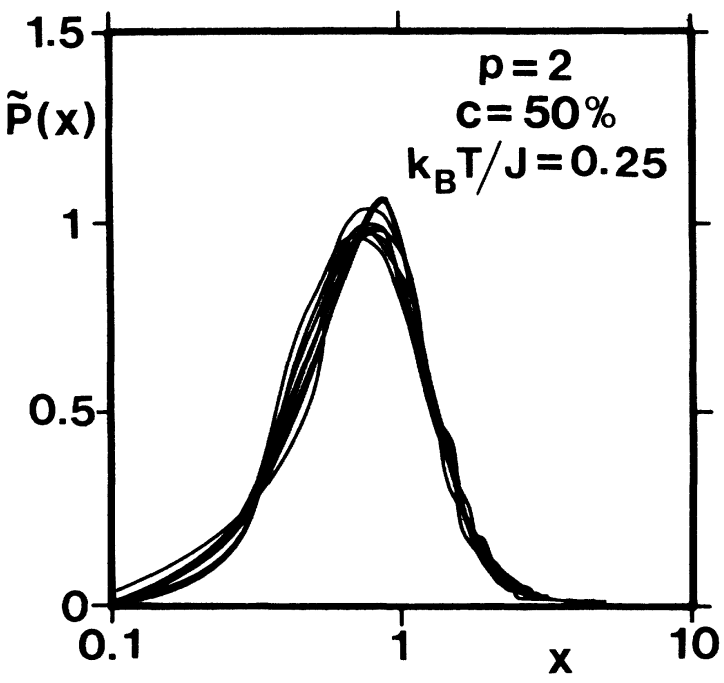

FIG. 15. Same data as in Fig. 14 plotted in a semilogarithmic diagram to reveal the log-normal shape of the dynamical scaling function, $\tilde{P}(x)$, for the domain-size distribution.

and differences between the two models.

The pure system, $c=0$, has previously been studied rather extensively ${ }^{19,24,27}$ and is found to obey the AllenCahn growth law, and the structure factor is found to exhibit dynamical scaling. In Fig. 16 we deliver additional evidence for dynamical scaling in this model by presenting the scaling function, $\tilde{P}(x)$, for the domainsize distribution function. The data for $\bar{R}(t), \Delta E(t)$, and $L(t)$ in Figs. 17-19 for $c=0$ show that the algebraic growth law, Eq. (1) with $n=\frac{1}{2}$, is fulfilled.

We then turn to quenches of the dilute model. In Fig. 20 is shown the time evolution of microconfigurations for $c=5 \%$. The same general phenomena of vacancy

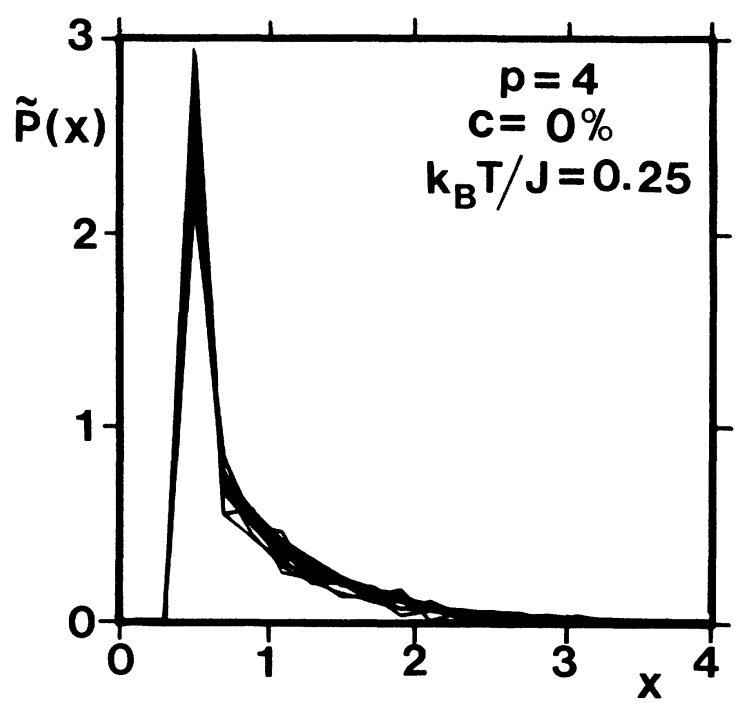

FIG. 16. Dynamical scaling function for the domain-size distribution function, $\tilde{P}(x)$ in Eq. (7), for quenches of the $p=4$ model at $c=0 \%$ to a temperature $k_{B} T / J=0.25$. Results are given for times $t$ in the range from 10 to 300 $\mathrm{MCS} / \mathrm{S}$ for a system with $200 \times 200$ sites. accumulation at the interfaces and precipitation inside the domains are observed during the fourfold-degenerate ordering process of the dilute system as for the $p=2$ model; cf. Figs. 8 and 12. As expected, the domain pattern is more compact the higher the ordering degeneracy is. Also in the case of the $p=4$ modei the dilution leads to a slowing down of the growth, as seen quantitatively in all three quantities, $\bar{R}(t), \Delta E(t)$, and $L(t)$; cf. Figs. 1719. The slowing down is most strongly signalled in the $\bar{R}(t)$ data. Replotting the $\bar{R}(t)$ data on a semilogarithmic scale, as demonstrated in Fig. 21, shows that also for the $p=4$ model there is, at late times, a crossover to an effectively logarithmic growth mode for the diluted system.

The dynamical scaling properties of the $p=4$ model are demonstrated in Fig. 22 which shows the domainsize distribution function, $P(R, t)$, and the corresponding scaling function, $\tilde{P}(x)$, at the temperature $k_{B} T / J=0.25$ for $c=5 \%$. Again we note that dynamical scaling is fulfilled over a substantial time range.

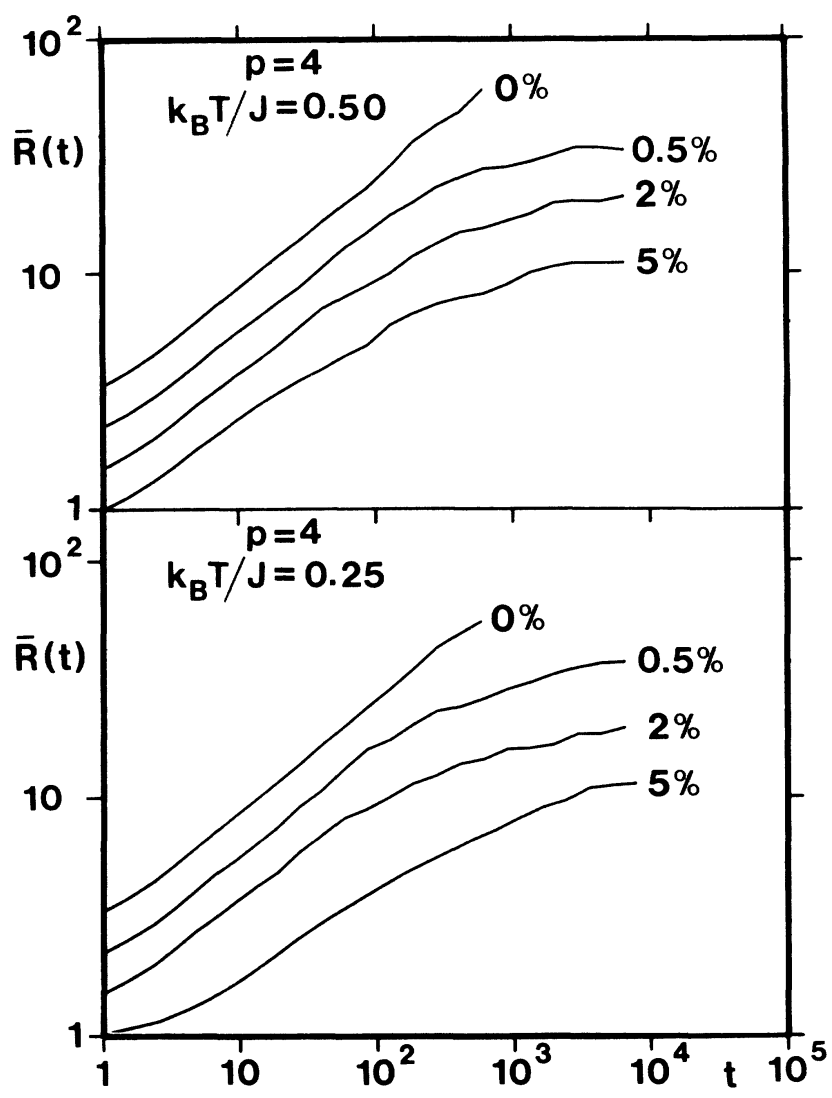

FIG. 17. Log-log plot of the average domain size, $\bar{R}(t)$, vs $t$ (in units of MCS/S) for quenches to two different tempertures, $k_{B} T / J=0.25$ and 0.50 , for different vacancy concentrations, $c$, in the $p=4$ dilute Ising model. For the sake of clarity, the various data sets have been appropriately translated along the vertical axis. 


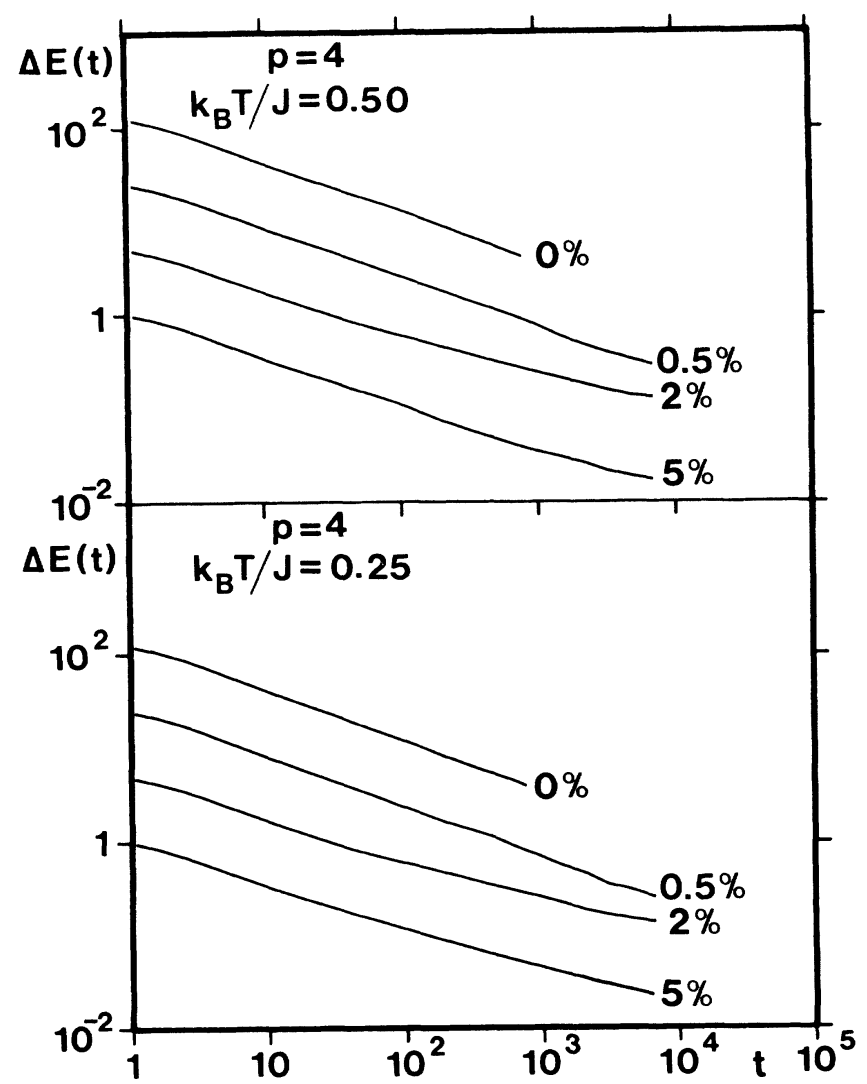

FIG. 18. Same as Fig. 17 but for the excess energy $\Delta E(t)$ in Eq. (5).

\section{COMPARISON WITH EXPERIMENT}

It is well known in materials science that impurities, vacancies and other types of imperfections have dramatic effects on grain-boundary mobility. ${ }^{75,76}$ Such effects are of a rather general nature and are observed also, e.g., in impure systems with solitons and discommensurates. ${ }^{77}$ As pointed out by Cahn ${ }^{75}$ in the case of grain growth, the important concepts in such problems are the grainboundary velocity relative to the diffusivity of the impu-

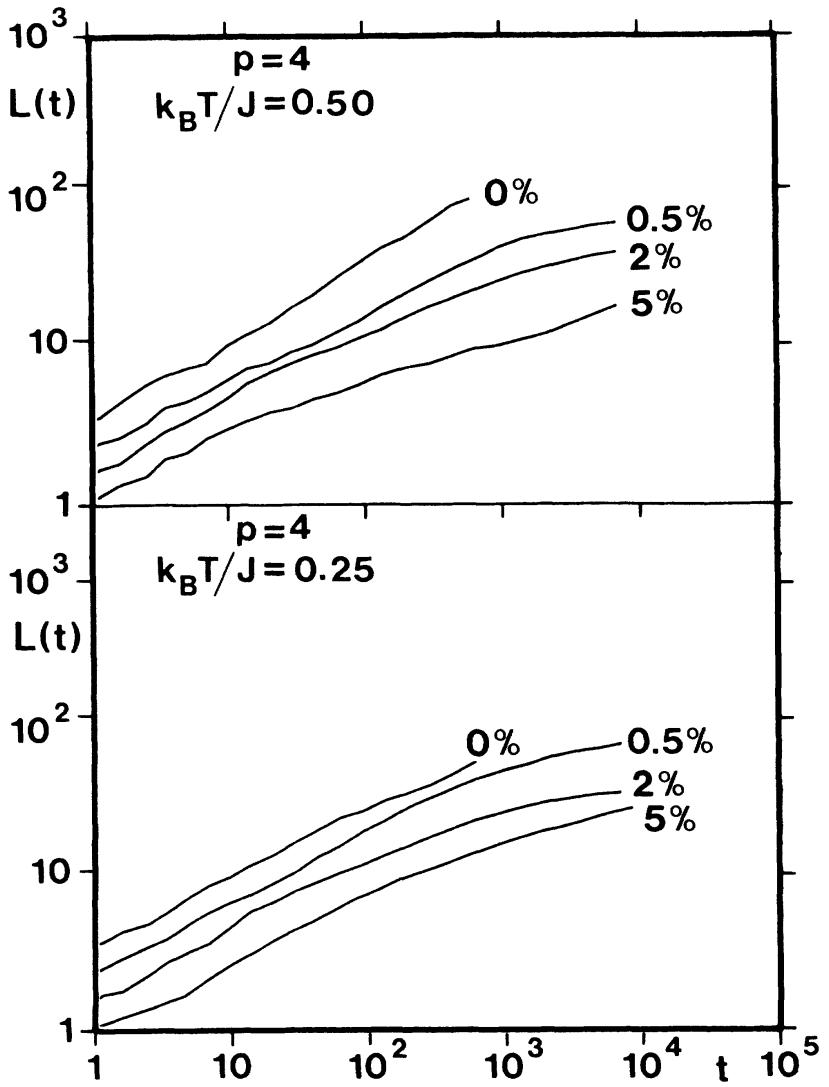

FIG. 19. Same as Fig. 17 but for the length measure $L(t)$ in Eq. (6).

rity (vacancy), as well as the impurity's interaction with the grain boundary: If the impurity dispersion is static, the growth will become pinned; if the impurity diffusivity is very fast compared to the grain-boundary velocity, the boundary mobility will not be affected by the impurities. This is also borne out by the computer-simulation study of the dilute Ising ferromagnet by Srolovitz and Hassold $^{46}$ who varied the vacancy diffusivity.

For real materials it is difficult to determine the precise relation between grain-boundary diffusion and vacancy

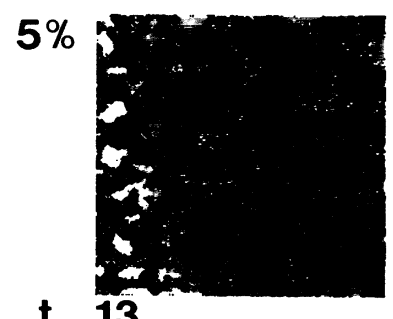

t 13

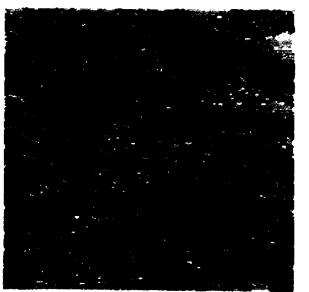

60

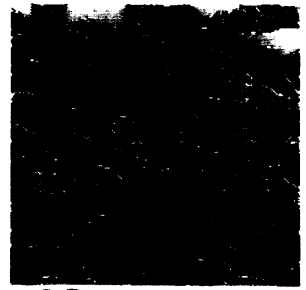

128

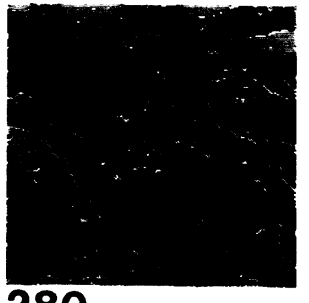

280

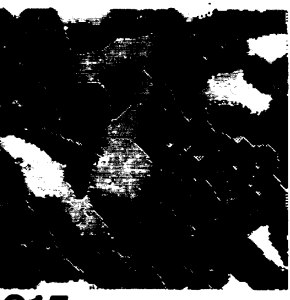

615

$$
p=4 \quad k_{B} T / J=0.25
$$

FIG. 20. Snapshots of microconfigurations for the $p=4$ model on a $200 \times 200$ lattice for $c=5 \%$ as they evolve in time $t$ (in units of MCS/S) for quenches to a temperature $k_{B} T / J=0.25$. The four types of antiferromagnetically ordered domains are indicated by black and three different grey-toned domains. 
or impurity diffusion, a situation which becomes further complicated by the fact that the diffusivity depends on temperature and hence varies during the quench. In the present model study we have restricted ourselves to the case where the boundary and the vacancy mobilities are comparable during the quench. Furthermore, the interactions between the vacancies and the boundary are chosen to be attractive; cf. Eq. (3). We believe that the general results of our study should apply, at least qualitatively, to a number of systems. Unfortunately, only a few systematic time-resolved experimental studies have been reported on the effect of mobile vacancies or impurities on the ordering dynamics of systems with order-disorder transitions. We shall here discuss our results in relation to these studies.

In a high-resolution LEED study Zuo et al. ${ }^{13}$ recently studied the ordering dynamics of an oxygen monolayer chemisorbed on W(112) doped with various amounts of nitrogen impurities. The symmetry of the oxygen ordering is that of the $p=2$ model of the present work. Zuo et al. ${ }^{13}$ found a distinct crossover from algebraic growth in the pure oxygen monolayer ${ }^{6,13}$ (described by the Allen-Cahn law) to a progressively slower growth mode as the impurity content is increased. This slow growth mode was, within the scatter of the data, found to be consistent with a logarithmic growth law, $\bar{R}(t) \sim \ln t$. Zuo et al. ${ }^{13}$ interpreted their finding within the theoret-

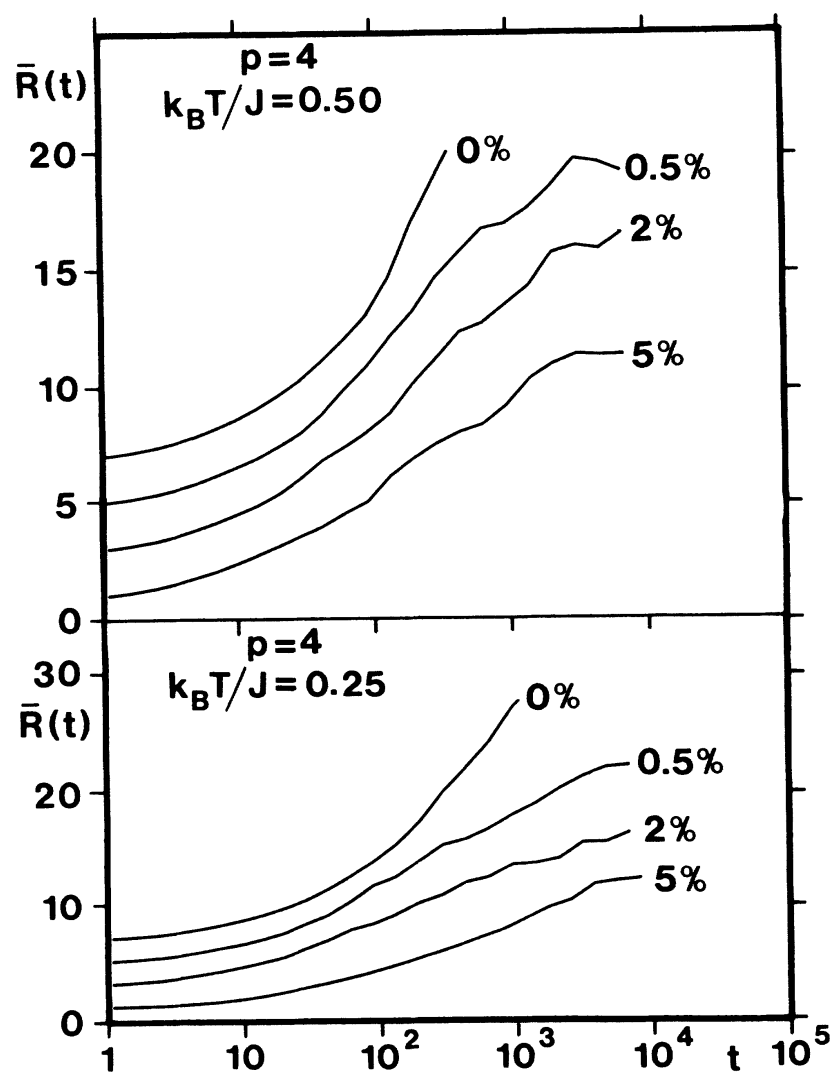

FIG. 21. Semilogarithmic plot of the data in Fig. 17.

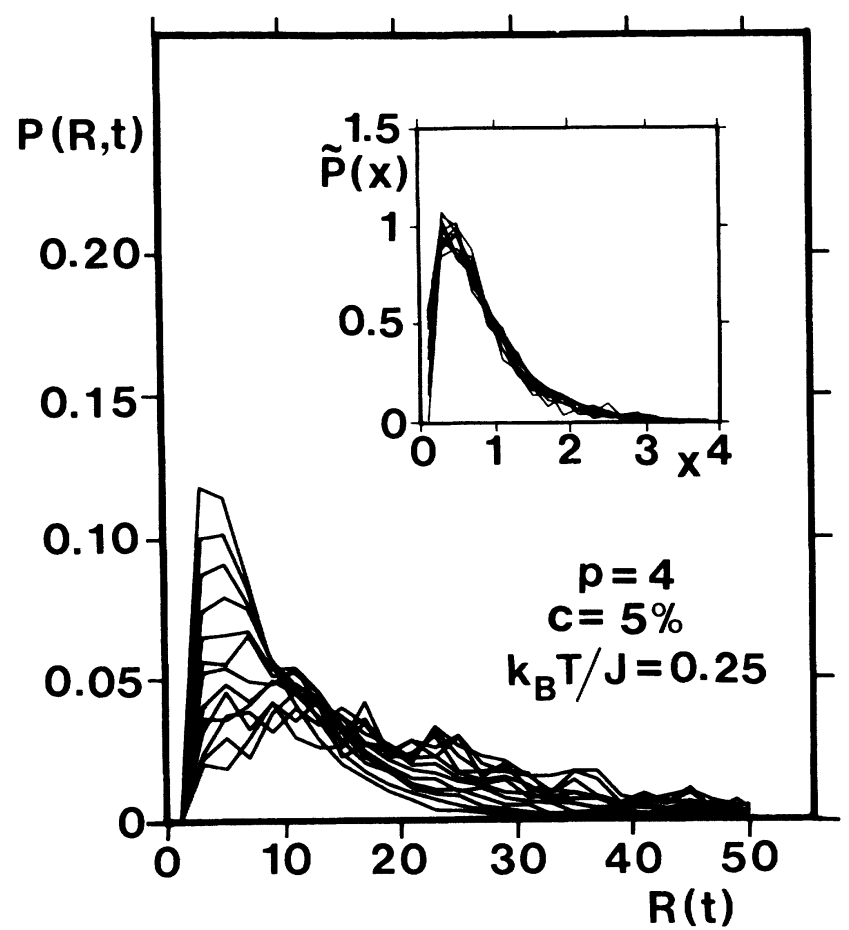

FIG. 22. Domain-size distribution function, $P(R, t)$, for quenches of the $p=4$ model to a temperature $k_{B} T / J=0.25$ for a vacancy concentration $c=5 \%$; cf. Fig. 20. The maximum of $P(R, t)$ moves to the right as time elapses. Results are given for the following times: $t=9,13,19,28,40,58$, $87,128,189,280,414,614$, and $911 \mathrm{MCS} / \mathrm{S}$. The inset shows the corresponding dynamic scaling function, $\tilde{P}(x)$ in Eq. (7), where $x=R(t) / \bar{R}(t)$ is the scaling variable.

ical framework of the random-field Ising model,${ }^{14}$ i.e., in terms of quenched randomness. However, as we have recently pointed out, ${ }^{64}$ surface diffusion data ${ }^{78}$ including prefactor and activation energy for $\mathrm{O}$ and $\mathrm{N}$ on tungsten surfaces in the pertinent temperature range (800-900 K) suggest that the diffusion of the nitrogen impurities is similar to that of oxygen. Therefore, our model predictions should apply when we interpret the mobile nitrogen impurities as a site dilution which does not participate in the oxygen ordering and does not couple directly to the order parameter (which is required by the randomfield Ising model). ${ }^{42}$ Therefore, we reinterpret the LEED results as a manifestation of slow, possible logarithmic growth, due to annealed randomness.

The ordering dynamics of oxygen on W(110), which has the symmetry of the $p=4$ model, has not been studied systematically in the presence of impurities. The experimentally observed late-time slowing down of the growth in supposedly pure oxygen monolayers has been ruled out experimentally as being due to impurities. ${ }^{7}$ In the light of the results of the present work, it should be pointed out that any analysis of impurity-controlled ordering in $\mathrm{O} / \mathrm{W}(110)$ should consider the possibility (depending on the O-impurity interactions as well as impurity-impurity interactions) that the mobile impurities may decouple the nearest-neighbor $\mathrm{O}-\mathrm{O}$ interactions 
and form a $(2 \times 2)$ superstructure.

Ordering processes in quenched off-stoichiometric alloys constitute a different class of systems in which diffusion of excess material may take the role of diffusion of impurities and vacancies. In these systems the ordering dynamics has to cope with the excess atoms. A particularly beautiful experimental demonstration of this phenomenon was recently reported by Shannon et al. ${ }^{14}$ who studied by time-resolved x-ray scattering the ordering dynamics in $\mathrm{Cu}_{0.79} \mathrm{Au}_{0.21}$ alloys. These authors found that the extra $\mathrm{Cu}$ gives rise to a crossover from an algebraic growth law (with the Allen-Cahn exponent $n=\frac{1}{2}$ ) in the (pure) stoichiometric system, ${ }^{10,14}\left(\mathrm{Cu}_{3} \mathrm{Au}\right)$, to a logarithmic growth law off stoichiometry; cf. Eq. (2) with $m=1-2$. Furthermore, Shannon et al. ${ }^{14}$ suggested picturing the ordering process as one in which the extra $\mathrm{Cu}$ precipitates into the antiphase domain boundaries. Since we do not expect the findings of the present paper to depend on the spatial dimension, we note that the experimental findings for the ordering dynamics in quenched off-stoichiometric alloys is in accordance with our computer-simulation results for the growth law. Furthermore, the picture put forward by Shannon et al. ${ }^{14}$ (see also Ref. 47) of accumulation of excess material in the domain boundaries is supported by our computersimulation snapshots; cf. Figs. 8 and 20 .

\section{CONCLUSIONS}

In this paper we have studied by Monte Carlo computer-simulation techniques the influence on the ordering dynamics of annealed dilution. Twofold $(p=2)$ as well as fourfold $(p=4)$ degenerate ordering has been studied. The ordering dynamics in the case of a nonconserved order parameter has been investigated as a function of temperature and degree of dilution. Our main result is that, independent of the temperature and the ordering degeneracy, there is a distinct crossover from an algebraic growth law, $\bar{R}(t) \sim t^{1 / 2}$, to a much slower growth mode as the system becomes diluted. This slower growth mode may be described as an effective logarithmic growth law, $\bar{R}(t) \sim \ln t$. These results are in accordance with recent experimental studies of the ordering dynamics in impure overlayers chemisorbed on solid substrates and in off-stoichiometric alloys.

Another important result is the finding of vacancy accumulation in the domain boundaries. As the vacancies accumulate in the domain boundaries, the direct domain-domain interactions become screened or even decoupled, implying that the curvature-driven pure-system Allen-Cahn growth mechanism becomes ineffective. This may provide a possible explanation of the logarithmic growth behavior in the dilute system, since the growth then proceeds via an evaporation-condensation mechanism which is an activated process, and the time it takes for two domains to merge depends on the size of the domains. Since the order parameter is not conserved in this process, the Lifshitz-Slyozov theory ${ }^{67}$ should not apply.
However, since we are dealing with a phase-separation problem between phases of different density, this theory may still be pertinent, ${ }^{79}$ i.e., $\bar{R}(t) \sim t^{1 / 3}$. We cannot exclude, on the basis of the present data, that the growth at late times crosses over to the Lifshitz-Slyozov law. It appears from our calculations that there may be two different morphological regimes for the ordering in annealed dilute systems, cf. Figs. 8 and 12 . For a very high dilution, e.g., $c=50 \%$, we are in a droplet regime, whereas for less dilution, $c=20 \%$, a convoluted percolation structure appears. These different regimes are similar to those observed in tricritical quenches in binary mixtures. ${ }^{47}$ Our results indicate that, whereas the growth behavior is effectively logarithmic in the percolative regime, within the droplet regime an effective algebraic growth law is effective with a rather low exponent, $n \simeq 0.16$. These statements seem to hold independent of temperature. Hence, it appears that a low density is not sufficient to relax the condition of conserved order parameter which is required for the Lifshitz-Slyozov evaporation-condensation mechanism to apply.

We emphasize that we have no theory for the two types of slow growth behavior in the dilute system and wish to remark that, although this behavior is also described by an effectively logarithmic growth law, it is not covered by either the theory of the random-field Ising model ${ }^{51,52}$ or the theory by Huse and Henley ${ }^{42}$ for a quenched dilution.

For both types of ordering degeneracies our results show, for both the pure and the dilute systems, that the domain-size distribution function (which is approximately log-normal) obeys dynamical scaling over a substantial time range. On the other hand, the different measures of length scale, specifically the average domain size $\bar{R}(t)$ and excess energy $\Delta E(t)$, no longer obey the relation $^{19} \bar{R}(t) \sim \Delta E^{-1}(t)$. This implies that $\Delta E^{-1}(t)$ is not a reliable measure of the total domain perimeter density, which was also observed by Srolovitz and Hassold ${ }^{46}$ for the dilute Ising ferromagnet.

We finally wish to point out that the model proposed in the present paper for studying ordering processes in highly dilute systems with open boundaries may serve as the basis for more involved models of compaction processes in condensed matter. Specific problems involve annealing of radiation-damaged materials and sintering processes in metals and ceramics. Some preliminary work by Srolovitz et al. ${ }^{80}$ on high-p-state dilute Potts models has revealed the temporal evolution of grain-size and pore-size distribution functions in two dimensions.

\section{ACKNOWLEDGMENTS}

The authors wish to thank Anders Høst-Madsen for participation in the preliminary stages of this research project. It is a pleasure to thank David J. Srolovitz for stimulating discussions concerning the effect of vacancy diffusion on grain growth. This work was supported by the Danish Technical Research Council under Grant No. 16-4296.K and the Danish Natural Science Research Council under Grant No. 5.21.99.72. 
${ }^{1}$ For a recent comprehensive selection of articles on experimental and theoretical studies of the dynamics of ordering processes, see Dynamics of Ordering Processes in Condensed Matter, edited by S. Komura and H. Furukawa (Plenum, New York, 1988).

${ }^{2}$ J. D. Gunton, M. San Miguel, and P. S. Sahni, in Phase Transitions and Critical Phenomena, edited by C. Domb and J. L. Lebowitz (Academic, New York, 1983), Vol. 8, p. 269.

${ }^{3}$ K. Heinz, in Kinetics of Interface Reactions, edited by M. Grunze and H. J. Kreuzer (Springer-Verlag, New York, 1987), p. 202.

${ }^{4}$ G.-C. Wang and T.-M. Lu, Phys. Rev. Lett. 50, 2014 (1983).

${ }^{5}$ P. K. Wu, J. H. Perepezko, J. T. McKinney, and M. G. Lagally, Phys. Rev. Lett. 51, 1577 (1983).

${ }^{6}$ M. C. Tringides, P. K. Wu, and M. G. Lagally, Phys. Rev. Lett. 59, 315 (1987).

${ }^{7}$ P. K. Wu, M. C. Tringides, and M. G. Lagally, Phys. Rev. B 39, 7595 (1989).

${ }^{8}$ S. Komura, K. Osamura, H. Fujii, and T. Takeda, Phys. Rev. B 31, 1278 (1985).

${ }^{9}$ S. Katano, M. Iizumi, R. M. Nicklow, and H. R. Child, Phys. Rev. B 38, 2659 (1988).

${ }^{10}$ S. E. Nagler, R. F. Shannon, C. R. Harkless, and M. A. Singh, Phys. Rev. Lett. 61, 718 (1988).

${ }^{11}$ D. P. Belanger, A. R. King, and V. Jaccarino, Phys. Rev. B 31, 4538 (1985).

${ }^{12}$ H. Yoshizawa, R. A. Cowley, G. Shirane, and R. Birgeneau, Phys. Rev. B 31, 4548 (1985).

${ }^{13}$ J.-K. Zuo, G.-C. Wang, and T.-M. Lu, Phys. Rev. Lett. 60, 1053 (1988).

${ }^{14}$ R. F. Shannon, C. R. Harkless, and S. E. Nagler, Phys. Rev. B 38, 9327 (1988).

${ }^{15}$ M. K. Phani, J. L. Lebowitz, M. H. Kalos, and O. Penrose, Phys. Rev. B 45, 366 (1980).

${ }^{16}$ P. S. Sahni, G. Dee, J. D. Gunton, M. Phani, J. L. Lebowitz, and M. Kalos, Phys. Rev. B 24, 410 (1981).

${ }^{17}$ P. S. Sahni and J. D. Gunton, Phys. Rev. Lett. 47, 1754 (1981).

${ }^{18}$ K. Kaski, M. C. Yalabik, J. D. Gunton, and P. S. Sahni, Phys. Rev. B 28, 5263 (1983).

${ }^{19}$ A. Sadiq and K. Binder, J. Stat. Phys. 35, 517 (1984).

${ }^{20}$ G. S. Grest and D. J. Srolovitz, Phys. Rev. B 30, 5150 (1984).

${ }^{21}$ E. T. Gawlinski, M. Grant, J. D. Gunton, and K. Kaski, Phys. Rev. B 31, 281 (1985).

${ }^{22}$ J. Viñals and J. D. Gunton, Surf. Sci. 157, 473 (1985).

${ }^{23}$ A. Milchev, K. Binder, and D. W. Heermann, Z. Phys. B 61, 521 (1986).

${ }^{24}$ A. Høst-Madsen, P. J. Shah, T. V. Hansen, and O. G. Mouritsen, Phys. Rev. B 36, 2333 (1987).

${ }^{25}$ S. Ohta, T. Ohta, and K. Kawasaki, Physica 140A, 478 (1987).

${ }^{26}$ J. G. Amar, F. E. Sullivan, and R. D. Mountain, Phys. Rev. B 37, 196 (1988).

${ }^{27}$ H. C. Fogedby and O. G. Mouritsen, Phys. Rev. B 37, 5962 (1988).

${ }^{28}$ Z. W. Lai, G. F. Mazenko, and O. T. Valls, Phys. Rev. B 37, 9481 (1988).

${ }^{29}$ J. Viñals and D. Jasnow, Phys. Rev. B 37, 9582 (1988).

${ }^{30}$ G. F. Mazenko, O. T. Valls, and M. Zannetti, Phys. Rev. B 38, 520 (1988).
${ }^{31}$ O. G. Mouritsen and E. Præstgaard, Phys. Rev. B 38, 2703 (1988)

${ }^{32}$ G. S. Grest, M. P. Anderson, and D. J. Srolovitz, Phys. Rev. B 38, 4752 (1988).

${ }^{33}$ A. Chakrabarti, J. B. Collins, and J. D. Gunton, Phys. Rev. B 38, 6894 (1988).

${ }^{34}$ T. Ala-Nissila and J. D. Gunton, Phys. Rev. B 38, 11418 (1988).

${ }^{35}$ H. C. Kang and W. H. Weinberg, Phys. Rev. B 38, 11543 (1988).

${ }^{36}$ O. G. Mouritsen, H. C. Fogedby, and E. Præstgaard, in $D y-$ namics of Ordering Processes in Condensed Matter, Ref. 1, p. 133.

${ }^{37}$ S. A. Safran, P. S. Sahni, and G. S. Grest, Phys. Rev. B 28, 2693 (1983); P. S. Sahni, D. J. Srolovitz, G. S. Grest, M. P. Anderson, and S. A. Safran, ibid. 28, 2705 (1983).

${ }^{38} \mathrm{~J}$. Viñals and M. Grant, Phys. Rev. B 36, 7036 (1987).

${ }^{39}$ D. J. Srolovitz, M. P. Anderson, G. S. Grest, and P. S. Sahni, Acta Metall. 32, 1429 (1984).

${ }^{40}$ G. S. Grest and D. J. Srolovitz, Phys. Rev. B 32, 3014 (1985).

${ }^{41}$ D. J. Srolovitz and G. S. Grest, Phys. Rev. B 32, 3021 (1985).

${ }^{42}$ D. A. Huse and C. L. Henley, Phys. Rev. Lett. 54, 2708 (1985).

${ }^{43}$ D. Chowdhury, M. Grant, and J. D. Gunton, Phys. Rev. B 35, 6792 (1987).

${ }^{44}$ O. G. Mouritsen, Phys. Rev. B 32, 1632 (1985).

${ }^{45}$ G. F. Mazenko and O. T. Valls, Phys. Rev. B 33, 1823 (1986).

${ }^{46}$ D. J. Srolovitz and G. N. Hassold, Phys. Rev. B 35, 6902 (1987).

${ }^{47}$ T. Ohta, K. Kawasaki, A. Sato, and Y. Enomoto, Phys. Lett. A 126, 93 (1987).

${ }^{48}$ J. H. Oh and D.-I. Choi, Phys. Rev. B 33, 3448 (1986).

${ }^{49}$ A. E. Jacobs and C. M. Coram, Phys. Rev. B 36, 3844 (1987).

${ }^{50}$ M. Grant and J. D. Gunton, Phys. Rev. B 29, 1521 (1984).

${ }^{51}$ J. Villain, Phys. Rev. Lett. 52, 1543 (1984).

${ }^{52}$ G. Grinstein and J. F. Fernandez, Phys. Rev. B 29, 6389 (1984).

${ }^{53}$ E. T. Gawlinski, K. Kaski, M. Grant, and J. D. Gunton, Phys. Rev. Lett. 53, 2266 (1984).

${ }^{54}$ E. T. Gawlinski, S. Kumar, M. Grant, J. D. Gunton, and K. Kaski, Phys. Rev. B 32, 1575 (1985).

${ }^{55}$ S. R. Anderson and G. F. Mazenko, Phys. Rev. B 33, 2007 (1986).

${ }^{56}$ D. Chowdhury and J. D. Gunton, J. Phys. A 19, L1105 (1986).

${ }^{57}$ M. Grant and J. D. Gunton, Phys. Rev. B 35, 4922 (1987).

${ }^{58}$ D. A. Huse, Phys. Rev. B 36, 5383 (1987).

${ }^{59}$ S. R. Anderson, Phys. Rev. B 36, 8435 (1987).

${ }^{60}$ M. Hillert, Acta Metall. 36, 3177 (1988).

${ }^{61}$ R. B. Stinchcombe, in Phase Transitions and Critical Phenomena, edited by C. Domb and M. S. Green (Academic, New York, 1983), Vol. 8, p. 151.

${ }^{62}$ P. C. Hohenberg and B. I. Halperin, Rev. Mod. Phys. 49, 435 (1977)

${ }^{63} \mathrm{~J}$. D. Gunton, in Kinetics of Interface Reactions, edited by M. Grunze and H. J. Kreuzer (Springer-Verlag, New York, 1987), p. 238.

${ }^{64}$ O. G. Mouritsen and P. J. Shah, Phys. Rev. B 40, 11445 (1989). 
${ }^{65}$ I. M. Lifshitz, Zh. Eksp. Teor. Fiz. 42, 1354 (1962) [Sov. Phys.-JETP 15, 939 (1962)].

${ }^{66}$ S. M. Allen and J. W. Cahn, Acta Metall. 27, 1085 (1979).

${ }^{67}$ I. M. Lifshitz and V. V. Slyozov, J. Phys. Chem. Solids 19, 35 (1961).

${ }^{68}$ K. Binder and D. P. Landau, Phys. Rev. B 21, 1941 (1980).

${ }^{69}$ O. G. Mouritsen, Computer Studies of Phase Transitions and Critical Phenomena (Springer-Verlag, New York, 1984).

${ }^{70}$ K. Binder and D. Stauffer, Phys. Rev. Lett. 33, 1006 (1974).

${ }^{71}$ H. A. Ceccatto, Phys. Rev. B 33, 4734 (1986).

${ }^{72}$ A. N. Burkitt and D. W. Heermann, Europhys. Lett. (to be published).

${ }^{73}$ R. H. Swendsen and J. S. Wang, Phys. Rev. Lett. 58, 86
(1987); U. Wolff, ibid. 62, 361 (1989).

${ }^{74}$ S. K. Kurtz and F. M. A. Carpay, J. Appl. Phys. 51, 5725 (1980); C. S. Pande, Acta Metall. 36, 2161 (1988).

${ }^{75}$ J. W. Cahn, Acta Metall. 10, 789 (1962).

${ }^{76}$ M. Hillert and B. Sundman, Acta Metall. 24, 731 (1976).

${ }^{77}$ D. J. Srolovitz, R. Eykholt, D. M. Barnett, and J. P. Hirth, Phys. Rev. B 35, 6107 (1987).

${ }^{78}$ A. Polak and G. Ehrlich, J. Vac. Sci. Technol. 14, 407 (1977); A. G. Naumovets and Y. S. Vedula, Surf. Sci. Rep. 4, 365 (1985).

${ }^{79}$ W. W. Mullins and J. Viñals, Acta Metall. 37, 991 (1989).

${ }^{80}$ W. M. Visscher, G. N. Hassold, and D. J. Srolovitz, Bull. Am. Phys. Soc. 33, 304 (1988); D. J. Srolovitz (private communication). 

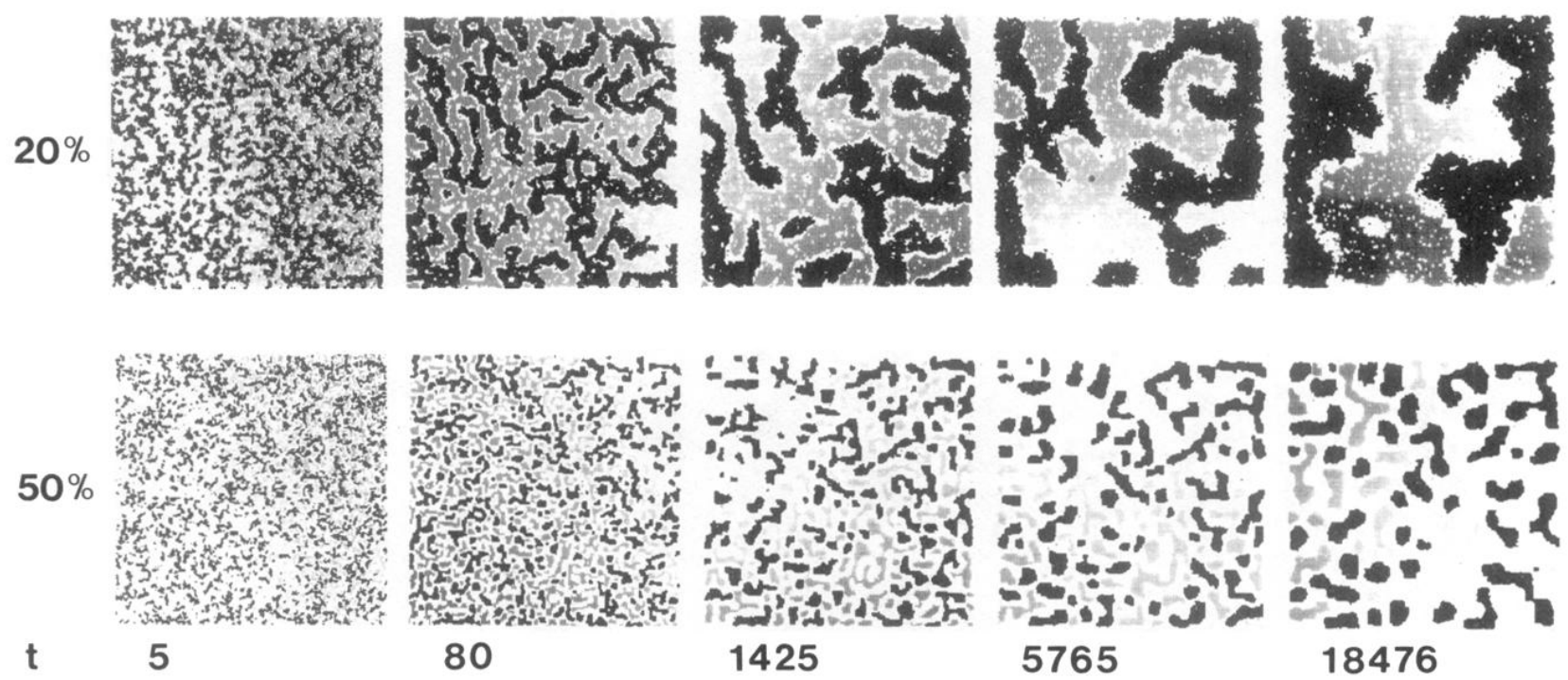

1425

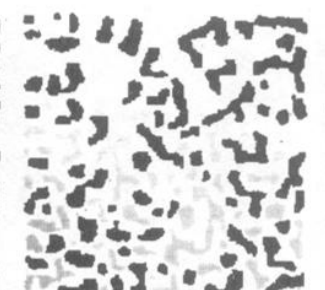

5765

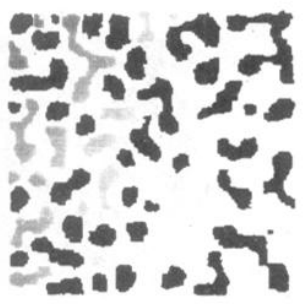

$$
p=2
$$

18476

FIG. 12. Snapshots of microconfigurations for the $p=2$ model on a $200 \times 200$ lattice as they evolve in time $t$ (in units of $\mathrm{MCS} / \mathrm{S}$ ) for quenches to a temperature $k_{B} T / J=0.50$ for $c=20 \%$ and a temperature $k_{B} T / J=0.25$ for $c=50 \%$. The two types of antiferromagnetically ordered domains are indicated by grey and black regions. 


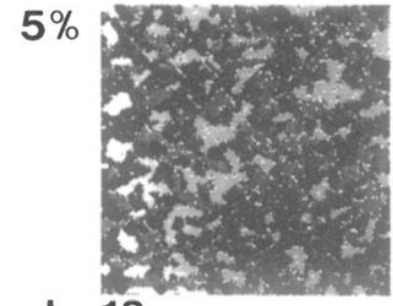

t 13

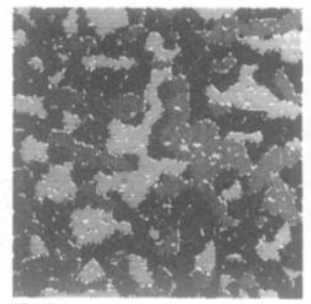

60

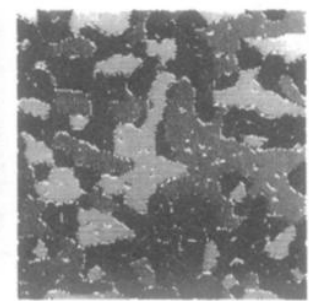

128

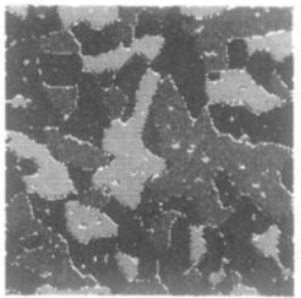

280

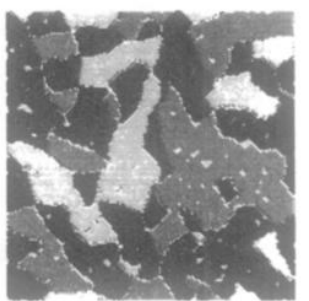

615

$$
p=4 \quad k_{B} T / J=0.25
$$

FIG. 20. Snapshots of microconfigurations for the $p=4$ model on a $200 \times 200$ lattice for $c=5 \%$ as they evolve in time $t$ (in units of $\mathrm{MCS} / \mathrm{S}$ ) for quenches to a temperature $k_{B} T / J=0.25$. The four types of antiferromagnetically ordered domains are indicated by black and three different grey-toned domains. 


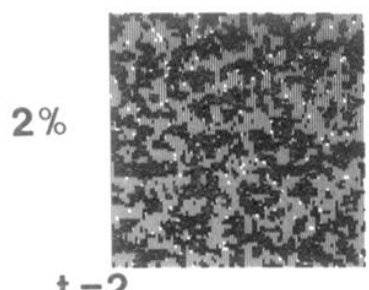

$t=2$
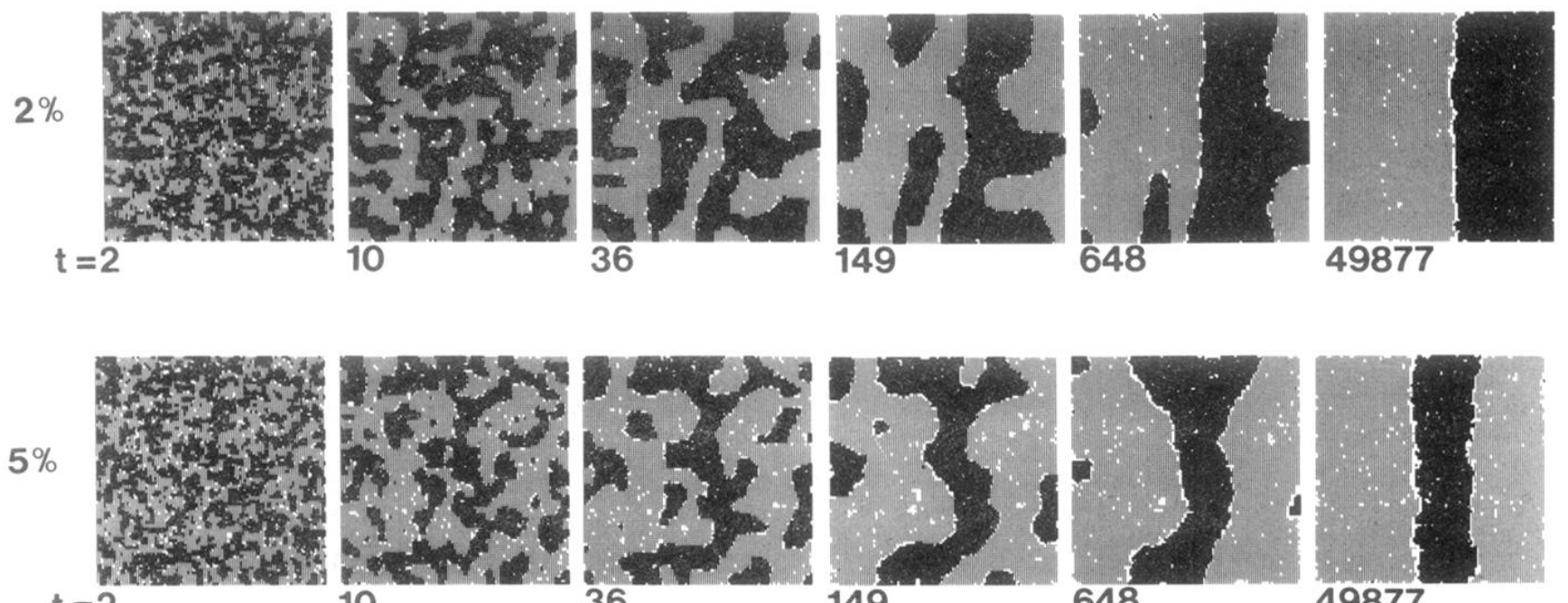

10

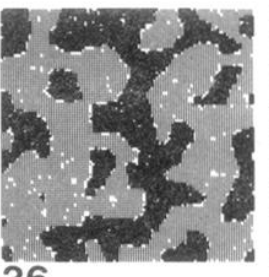

36

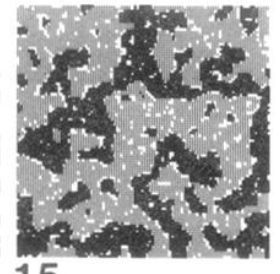

15

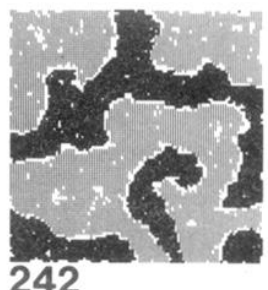

242

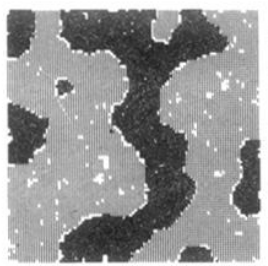

149
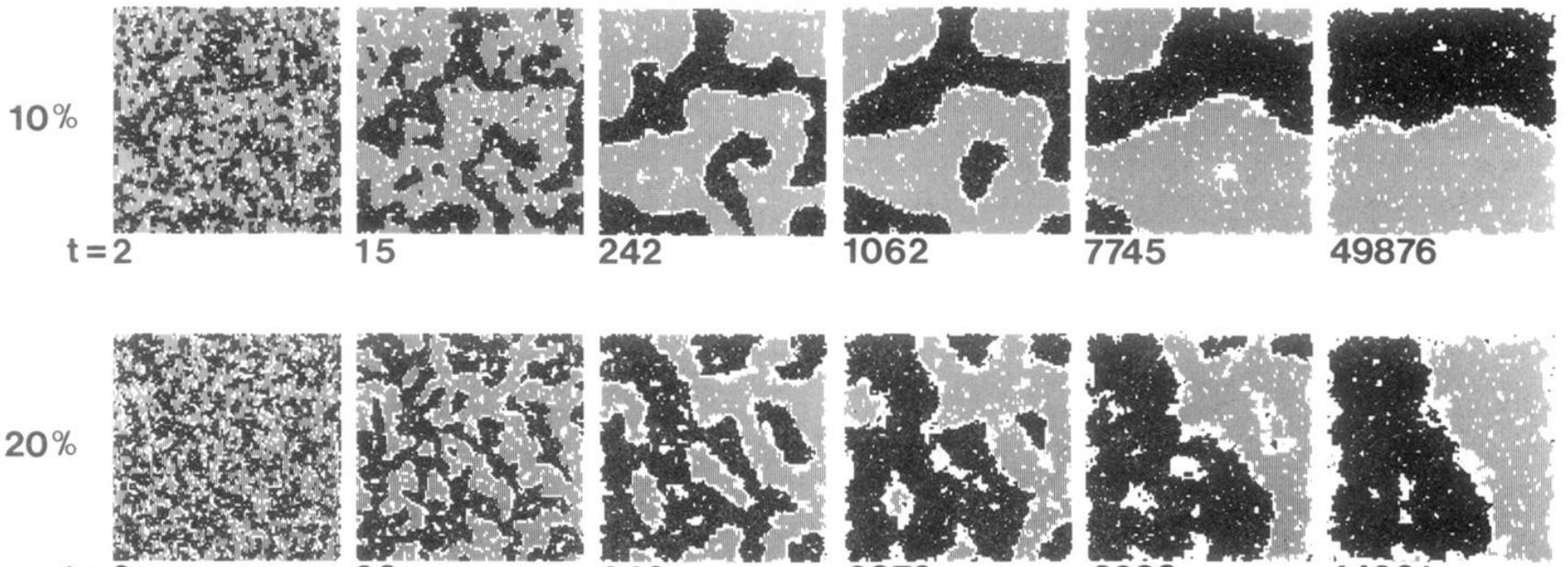

$t=2$

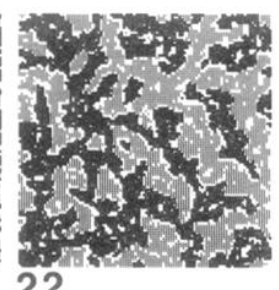

22
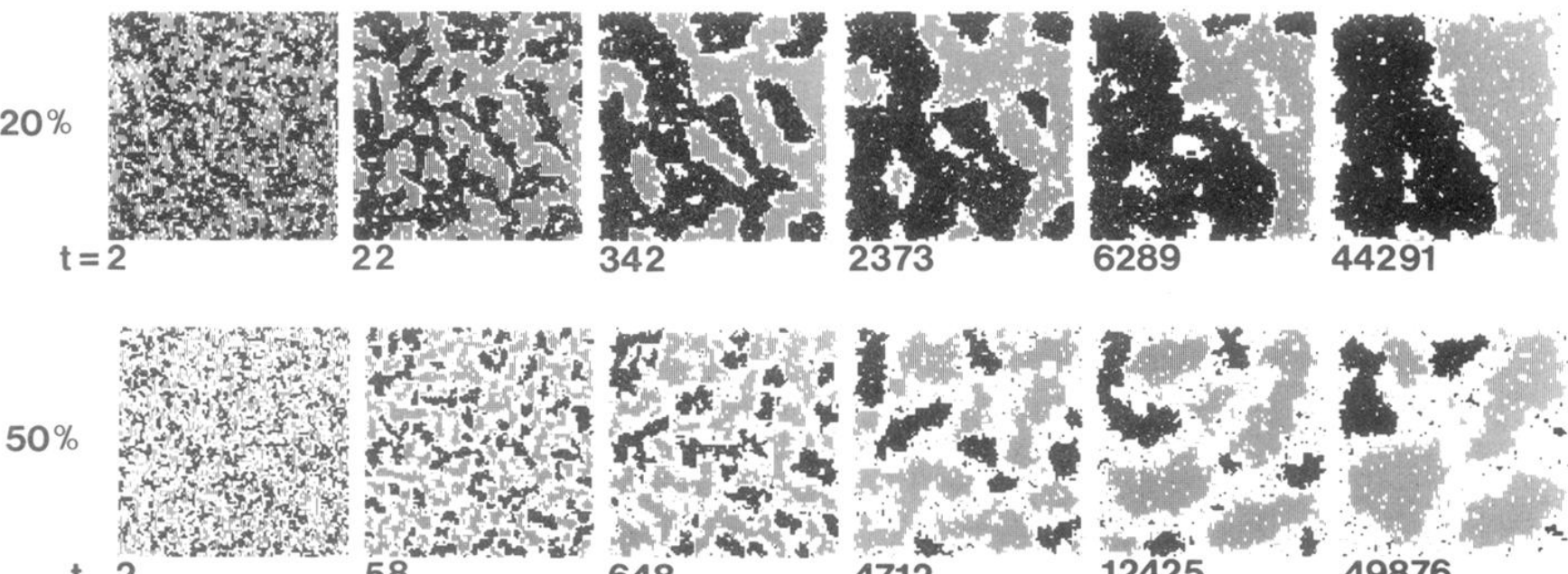

t 2

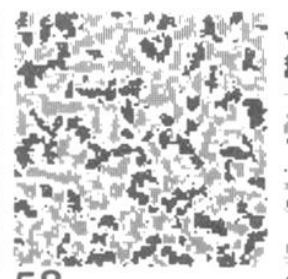
58

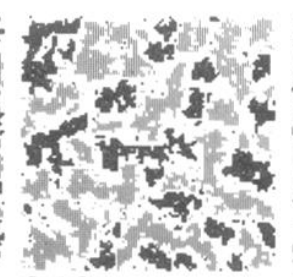

648

$$
\mathrm{p}=2 \quad \mathrm{k}_{\mathrm{B}} \mathrm{T} / \mathrm{J}=0.50
$$
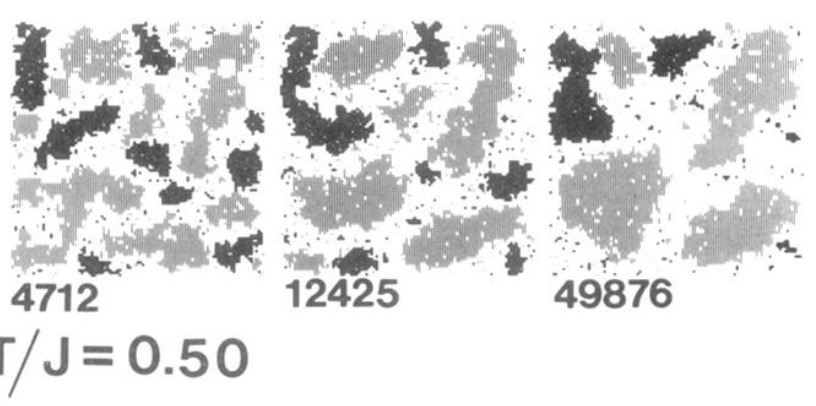

FIG. 8. Snapshots of microconfigurations for the $p=2$ model as they evolve in time $t$ (in units of MCS/S) after quenches to a temperature $k_{B} T / J=0.50$ for vacancy concentrations $c=2 \%, 5 \%, 10 \%, 20 \%$, and $50 \%$. The two types of antiferromagnetically ordered domains are indicated by grey and black regions. The snapshots refer to lattices with $100 \times 100$ sites. 

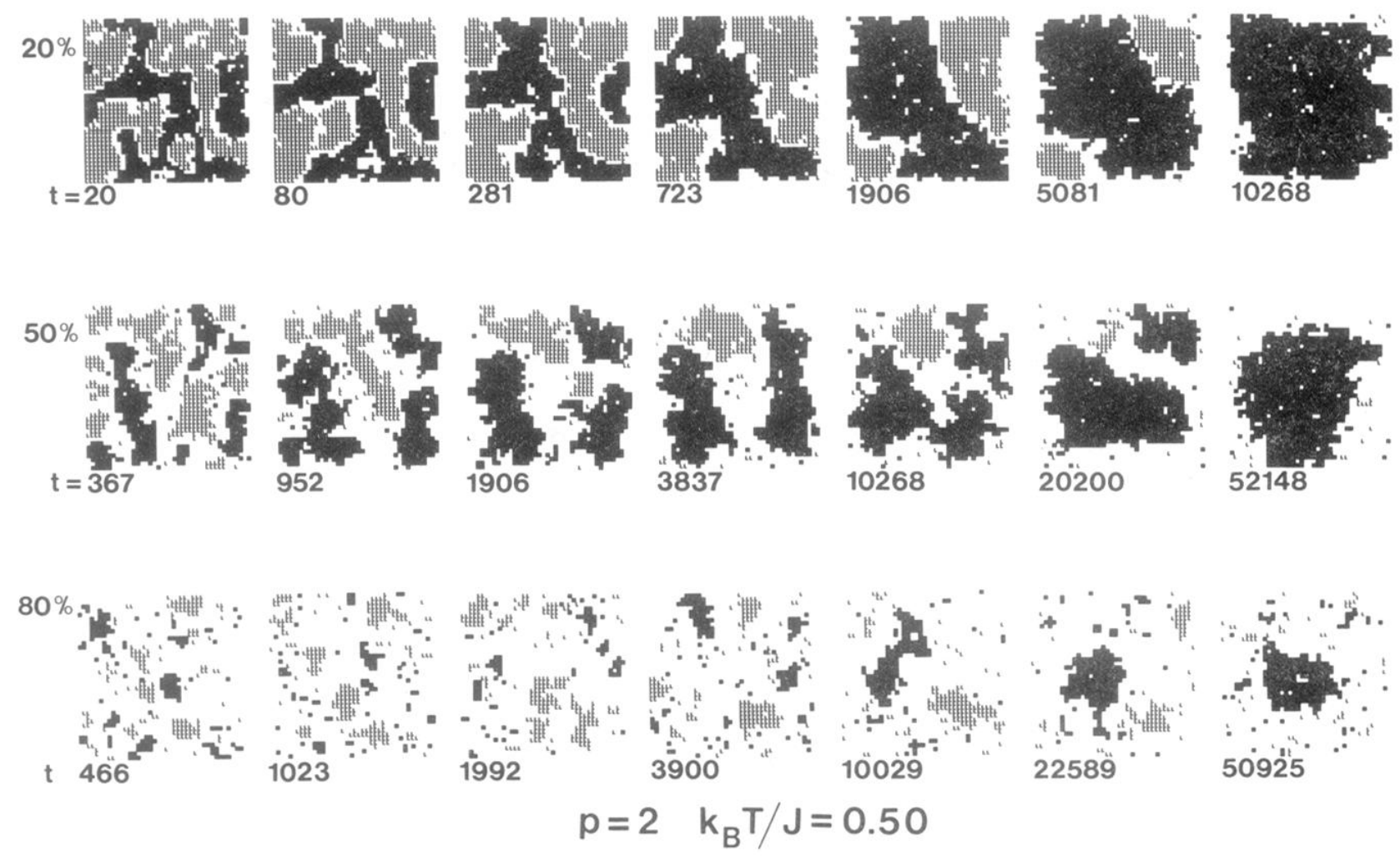

FIG. 9. Snapshots of microconfigurations for the $p=2$ model as they evolve in time $t$ (in units of MCS/S) into the equilibrium compact state after quenches to a temperature $k_{B} T / J=0.50$ for high vacancy concentrations, $c=20 \%, 50 \%$, and $80 \%$. The two types of order are indicated by grey and back regions. The configurations refer to a lattice with $40 \times 40$ sites. 